\author{
Matthijs Oudkerk \\ Arthur E. Stillman \\ Sandra S. Halliburton \\ Willi A. Kalender \\ Stefan Möhlenkamp \\ Cynthia H. McCollough \\ Rozemarijn Vliegenthart \\ Leslee J. Shaw \\ William Stanford \\ Allen J. Taylor \\ Peter M. A. van Ooijen \\ Lewis Wexler \\ Paolo Raggi
}

Received: 17 March 2008

Revised: 5 May 2008

Accepted: 19 May 2008

Published online: 24 July 2008

(C) The Author(s) 2008

This consensus article is being published concurrently in the Springer Journal International Journal of Cardiovascular Imaging.

M. Oudkerk · R. Vliegenthart

P. M. A. van Ooijen $(\bowtie)$

Department of Radiology,

Groningen University Hospital,

Hanzeplein 1,

9700 RB Groningen, The Netherlands

e-mail: p.m.a.van.ooyen@rad.azg.nl

A. E. Stillman · L. J. Shaw · P. Raggi

Department of Radiology,

Emory University,

Atlanta, GA, USA

\author{
A. E. Stillman - L. J. Shaw · P. Raggi \\ Department of Cardiology, \\ Emory University, \\ Atlanta, GA, USA
}

\section{Coronary artery calcium screening: current status and recommendations from the European Society of Cardiac Radiology and North American Society for Cardiovascular Imaging}

S. S. Halliburton

Imaging Institute, Cardiovascular

Imaging Lab, Cleveland Clinic

Foundation,

Cleveland, $\mathrm{OH}$, USA

\section{W. A. Kalender}

Institute of Medical Physics at the

Friedrich-Alexander-University

Erlangen-Nürnberg,

Nürnberg, Germany

S. Möhlenkamp

West German Heart Center Essen,

University Duisburg-Essen,

Essen, Germany

C. H. McCollough

Department of Radiology, Mayo Clinic,

Rochester, MN, USA

W. Stanford

Department of Radiology,

University of Iowa,

Iowa City, IA, USA

\author{
A. J. Taylor \\ Division of Cardiology, \\ Walter Reed Army Medical Center, \\ Washington, DC, USA
}

\author{
L. Wexler \\ Department of Radiology, \\ Stanford University School of Medicine, \\ Stanford, CA, USA
}

\begin{abstract}
Current guidelines and literature on screening for coronary artery calcium for cardiac risk assessment are reviewed for both general and special populations. It is shown that for both general and special populations a zero score excludes most clinically relevant coronary artery disease. The importance of standardization of coronary artery calcium measurements by multidetector $\mathrm{CT}$ is discussed.
\end{abstract}

Keywords Coronary artery calcium . Coronary artery atherosclerosis . Coronary risk assessment . Coronary artery CT

\section{Introduction}

In 1996 and 2000 the American Heart Association (AHA) issued statements on coronary artery calcium (CAC) quantification [1, 2]. In 2006 and 2007, several professional societies updated these statements describing new evidence related to CAC imaging [3-5]. The purpose of the present work is to summarize the rationale and content of those recommendations with regard to $\mathrm{CAC}$ quantification, to address differences among them, and to point out controversial areas of CAC research of high clinical relevance among both asymptomatic and symptomatic persons.
Nearly all of the published clinical outcome data from $\mathrm{CAC}$ are based on results obtained with electron beam tomography (EBT) systems. However, these CT systems are largely no longer available and are being widely replaced with multidetector CT (MDCT) systems. EBT, which was produced by one manufacturer, provided much more standardization than exists for all the various generations of MDCT systems from different manufacturers. Standardization guidelines have been proposed for MDCT [6] but are rarely used. Studies with earlier MDCT technology (4-16 slice) have demonstrated that similar mean CAC scores can be obtained with EBT and MDCT 
[7-12]. Nevertheless systematic differences exist that can likely affect serial measurements $[6,13,14]$.

While large numbers of patients were included in the EBT outcome studies, most of these studies suffered from selection biases related to ethnicity, patient self-referral, or referral by physicians concerned about subclinical coronary artery disease (CAD) due to the presence of risk factors. The Dallas Heart Study [15], Multi-Ethnic Study of Atherosclerosis [16], and Rotterdam Study [17, 18] have attempted to address some of these issues. The results from general populations cannot reliably be applied to special populations. Nevertheless it is clear from many studies that in all kinds of populations, even in high-risk populations such as diabetic patients, and in both symptomatic and asymptomatic patients, the absence of CAC (zero calcium score) excludes most clinically relevant CAD. This information is highly relevant since many individuals among the general as well as special populations have a zero calcium score.

In this paper we review the evidence in support of the use of CAC for cardiac risk assessment in the general population as well as special populations with particular focus on the importance of a zero score. The need for standardization of CAC measurements with MDCT is also discussed.

\section{Coronary artery calcium as a predictor of cardiac events}

Most published studies addressing the issue of CAC as a predictor of cardiac events are based on EBT data. In the ACCF/AHA Consensus Document on Coronary Artery Calcium Scoring, six such studies were selected for review because they fulfilled sufficient criteria for outcome analysis [18-23]. From the data presented in these studies it was concluded that CAC scores add incremental prognostic value in the evaluation of patients at intermediate risk for a coronary event. Other studies further support this conclusion. Raggi et al. [24] screened 632 asymptomatic patients with EBT and followed them for 32 months to determine the incidence of hard cardiac events (myocardial infarction and death). The majority of events occurred in individuals with high calcium scores and in individuals with scores $>75$ th percentile compared with age- and sex-matched controls. Arad et al. [19] screened 1,172 asymptomatic patients with EBT and followed them for a mean of 3.6 years to determine the incidence of cardiovascular endpoints (myocardial infarction, death, and the need for revascularization). The authors concluded that in asymptomatic adults, EBT calcium scores are highly predictive of events. Pletcher et al. [25] conducted a meta-analysis of studies performed between 1980-2003 in 13,000 asymptomatic patients screened with EBT and followed for 3.6 years or less to determine the odd ratios (OR) of hard coronary events. OR for Agatston CAC scores $<100,100-400$, and $>400$ were 2.1,
4.2 , and 7.2, respectively. The authors concluded that the EBT-derived Agatston calcium score is an independent predictor of coronary events in asymptomatic subjects.

\section{Coronary artery calcium as an indicator of coronary artery luminal stenosis}

Only studies based on EBT data have addressed the issue of CAC as an indicator of coronary artery luminal stenosis. Haberl et al. [26] analyzed the value of EBT-derived calcium CAC scores as an indicator of coronary luminal stenoses in 1,764 patients undergoing conventional coronary angiography. The authors concluded that EBT CAC scores are highly sensitive but moderately specific determinants of stenosis. The ACC/AHA Expert Consensus Document on Electron-Beam CT for the Diagnosis and Prognosis of Coronary Artery Disease [1] reported a pooled sensitivity of $91.8 \%$ and specificity of $55 \%$ for detection of a coronary artery stenosis $>50 \%$ after reviewing 16 selected studies comparing $\mathrm{CAC}$ scores and invasive angiography. Knez et al. [27] compared EBTderived Agatston CAC scores with calcium volume scores (CVS) as a predictor of coronary luminal stenoses in 2,115 patients undergoing conventional coronary angiography. The authors reported overall results similar to those already reported by others, but also concluded that the CVS is as accurate as the Agatston score for stenosis prediction. Indeed, Budoff et al. [28] utilized CVS obtained by EBT to predict the presence of coronary artery stenoses in 1,851 patients undergoing conventional coronary angiography and concluded that CVS provide incremental value in predicting the severity and extent of angiographically significant CAD.

\section{Clinical comparison of MDCT and EBT for coronary artery calcium score measurement}

Knez et al. [12] studied 99 symptomatic men (mean age: 60 years) with both MDCT (prospective triggering, Siemens Volume Zoom) and EBT imaging and found a correlation coefficient of 0.99 for CVS and 0.98 for the mass score (MS) with a mean overall variability of $17 \%$. No significant differences for scores 1-100, 101-400, 401$1000,>1000$ were found. The authors concluded that MDCT is equivalent to EBT for CAC scoring. Becker et al. [7] compared a 4-slice MDCT (Siemens Volume Zoom) with EBT (prospective triggering) in 100 patients and calculated the Agatston score, CVS, and MS. The authors concluded that the score variability is highest for the Agatston score (32\%), and the correlation between MSCT and EBT is excellent for CVS and MS. Carr et al. [8] performed CT examinations with both GE Lightspeed LX/i 4-slice MDCT (retrospective gating) and EBT in 36 patients and calculated the Agatston score in all of them. 
The authors reported excellent correlation between scores obtained on the two CT systems. Horiguchi et al. [11] performed EBT and 16-MDCT with retrospective gating in 100 patients and reported a high degree of correlation between the two CT systems for the Agatston score $\left(R^{2}=\right.$ $0.955)$, CVS $\left(R^{2}=0.952\right)$, and MS $\left(R^{2}=0.977\right)$. Daniell et al. [9] compared the results of EBT and 4-slice MDCT (prospective triggering, Siemens Volume Zoom) in 68 patients. EBT and MDCT scores correlated well $(R=0.98$ 0.99 ) with a median variability between EBT and MDCT for the Agatston score of $\sim 25 \%$ and $\sim 16 \%$ for CVS. Scores were higher for EBT than MDCT in approximately half of the cases, with little systematic difference between the two (median EBT-MDCT difference: Agatston score, -0.55 ; volume score, $3.4 \mathrm{~mm}^{3}$ ).

\section{Review of current guidelines on coronary artery calcification}

Risk assessment in asymptomatic persons

Risk stratification algorithms such as the Framingham risk score (FRS) [29], the PROCAM score [30], or the European SCORE system [31, 32] are used to assess an individual's global 10-year risk. Risk factors are measured and weighed and attributed to an empirically determined absolute risk of cardiovascular events, i.e., cardiac death and myocardial infarction: [33]

- Low risk $=<1 \%$ per year or $<10 \%$ in 10 years

- Intermediate risk $=1-2 \%$ per year or $10-20 \%$ in 10 years

- High risk $=>2 \%$ per year or $>20 \%$ in 10 years.

This classification was slightly modified by the 2004 update of NCEP guidelines (Table 1) [34].

It is argued that persons at high risk will most likely benefit from intensive risk modification, while persons at low risk are generally recommended to adhere to a healthy lifestyle and guideline-based treatment of individual risk factors when present. In persons at intermediate risk, however, there remains a diagnostic gap and further tests, such as CAC scoring, measuring intima-media thickness (IMT), the anklearm index, or exercise stress testing, may be useful in distinguishing individuals who indeed have a high risk from those at low risk, leaving hopefully few that remain at intermediate risk [33]. It should be recognized, however, that the Framingham score does not take into account lifestyle factors such as diet, exercise, and body mass index. Nor does the score reflect a positive family history of cardiovascular disease. The extent of atherosclerotic disease burden, autonomic dysfunction, chronic inflammation, lipoprotein subfractions, blood thrombogenicity, the myocardial propensity to develop life-threatening arrhythmias, and immeasurable genetic factors are also not part of conventional risk assessment. Measuring the atherosclerotic sequelae of life-long global exposure to all risk factors by virtue of measuring the extent of the disease in its early subclinical stages may overcome this limitation. The detection of calcified atherosclerosis is a general surrogate of total atheroma burden. It is noted however, that the extent of coronary calcification systematically differs among ethnic populations and by gender.

\section{The focus of current guidelines on CAC scoring}

The AHA Scientific Statement on "Assessment of Coronary Artery Disease (CAD) by Cardiac Computed Tomography" [3] reviewed scientific data for cardiac CT related to imaging of CAD and atherosclerosis in symptomatic and asymptomatic subjects, including a detailed description of technical aspects and radiation exposure of CAC CT and noninvasive $\mathrm{CT}$ angiography using electron beam $\mathrm{CT}$ (EBT) and multidetector CT (MDCT). According to AHA standards, recommendations were classified (Class I, IIa, $\mathrm{IIb}$, and III) and the level of evidence (A, B, or C) was provided (see http://circ.ahajournals.org/manual/manual_ IIstep6.shtml).

The ACC/AHA 2007 Clinical Expert Consensus Document [4] discussed the role of CAC quantification with respect to (1) identifying and modifying coronary event risk in asymptomatic subjects, (2) modifying clinical care and outcomes of symptomatic patients with suspected

Table 1 Absolute risk categories "NCEP Update 2004," modified from [34]

\begin{tabular}{ll}
\hline 10 -year risk categories & Definition of risk category \\
\hline High risk & $\mathrm{CAD},{ }^{\mathrm{a}}$ CAD-risk equivalents, ${ }^{\mathrm{b}} \geq 2$ major risk factors, ${ }^{\mathrm{c}}$ 10-year risk $>20 \%$ \\
Moderately high risk & $\geq 2$ major risk factors but 10 -year risk $<10 \%$ \\
Moderate risk & $\geq 2$ major risk factors but 10 -year risk $<10 \%$ \\
Low risk & $0-1$ major risk factor and 10 -year risk $<10 \%$
\end{tabular}

$C A D$ Coronary artery disease

${ }^{a}$ History of myocardial infarction, coronary revascularizations, or myocardial ischemia

${ }^{\mathrm{b}}$ Includes diabetes mellitus, stroke, TIA or carotid artery stenosis $>50 \%$, symptomatic peripheral artery disease or abdominal aortic aneurysm

${ }^{\mathrm{c}}$ Smoking, hypertension, high LDL-cholesterol/low HDL-cholesterol, age (men $>45$ years, women $>55$ years), and premature family history of CAD (1st-grade family member, i.e., men $<55$ years, women $<65$ years) 
CAD, and (3) understanding the role of CAC CT in selected patient sub-groups, including women, ethnic groups, and patients with renal disease or diabetes. 4) The clinical value of serial CAC CT, cost-effectiveness of CAC CT, and clinical implications of incidental findings were also addressed.

The purpose of the 2006 Appropriateness Criteria Statement [5] was to create, review, and categorize appropriateness criteria for cardiac CT and also cardiac magnetic resonance imaging (MRI) with regard to detection of CAD, cardiovascular risk stratification, as well as cardiac structure and function assessment. Members of the expert group assessed the risks and benefits of the imaging tests for several indications and clinical scenarios and scored them based on a scale of 1 to 9:

- 7 to $9=$ appropriate: the test is generally acceptable and is a reasonable approach

- 4 to $6=$ uncertain: uncertain indication or clinical setting

- 1 to 3 = inappropriate: the test is generally not acceptable/is not a reasonable approach

Indications in the latter statement were derived from common applications or anticipated uses of cardiac CT and MRI. Working group panelists rated each indication based on the ACC Methodology for Evaluating the Appropriateness of Cardiovascular Imaging [35].

\section{Indications for coronary artery calcium scoring in asymptomatic individuals}

In the past several years, numerous publications have reported on the incremental prognostic value of CAC over measured conventional risk factors in large series of patients including asymptomatic population-based cohorts $[18,20$ $23,36]$. The relative risk of coronary events increased with increasing CAC burden (Fig. 1). The majority of the expert writing committees agree that it may be reasonable to consider use of CAC measurement in asymptomatic intermediate risk patients, i.e., those at $10-20 \%$ risk of coronary events in

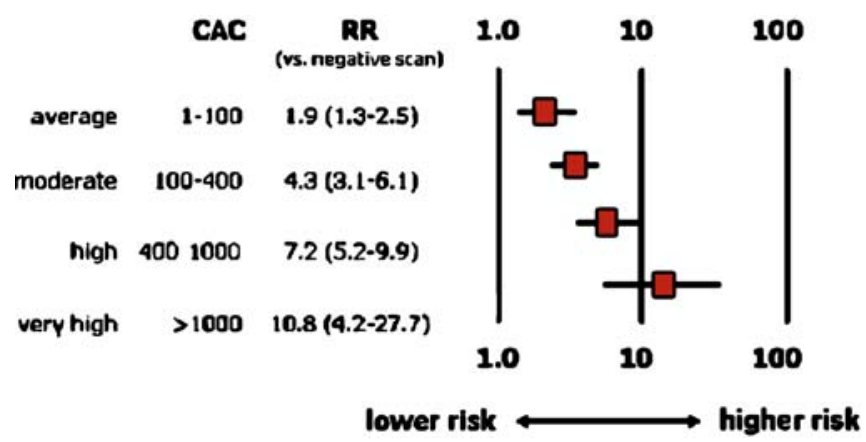

Fig. 1 Increase in relative risk (RR) with increasing CAC scores in asymptomatic persons in comparison to asymptomatic persons without CAC (modified from [20])
10 years. These patients might be reclassified to a higher risk status based on a high CAC score, and subsequent patient management may be modified (Fig. 2). In the ACC Appropriateness Criteria Document, notably published prior to the most recent AHA and ACC statements and additional prospective CAC scoring studies, CAC scoring was considered appropriate only for very few indications. However, all of them were given a rating of "uncertain," most notably CAC scoring for risk assessment in the general population at moderate (score 6) or high (score 5) CAD Framingham risk. In patients with a low or a high 10-year risk of coronary events, i.e., $<10 \%$ or $>20 \%$ risk in 10 years, CAC quantification is not recommended. In low risk individuals, even a high CAC score does not generally elevate this person's risk above the threshold to initiate therapy [4]. Yet, life-time risk may be elevated in 18 and $20 \%$ of asymptomatic men and women, respectively, who have a high CAC score, i.e., $\mathrm{CAC}>400$ or $>75$ th percentile, despite a low Framingham risk score [37]. Persons with a high 10-year risk are candidates for intensive risk modification based on current NCEP guidelines [34], and there is no evidence that a low CAC score substantially reduces this risk. This also holds for persons with risk equivalents. The performance of serial (follow-up) calcium scoring examinations was not recommended.

\section{Atherosclerotic disease quantification in patients with chest pain}

In symptomatic patients, diagnostic tests may be used for risk stratification, but the primary initial objective is to identify or rule out obstructive CAD. Especially in young persons with atypical chest pain, nonatherosclerotic nonobstructive coronary disease, such as myocardial bridging, coronary anomalies, coronary vasospasm, intramyocardial small vessel disease, or noncoronary heart disease such as cardiomyopathy, valvular heart disease, pericardial disease, aortic disease, and pulmonary disease, must be considered as differential diagnoses. Persons with an intermediate pretest likelihood of obstructive CAD, i.e., between $20-80 \%$, are most likely to benefit from additional testing [38].

Functional tests such as treadmill, exercise, or pharmacological nuclear stress tests or stress echocardiography are used to induce myocardial ischemia in patients with flowlimiting coronary obstruction. In contrast, CAC CT is aimed at estimating coronary plaque severity and the associated likelihood of a flow-limiting lesion. These two approaches, the former functional and the latter morphological, are distinctly different and have inherently different reasons for false-positive and false-negative results.

The presence of CAC is almost $100 \%$ sensitive for the presence of atherosclerotic coronary plaque but not specific for flow-limiting plaque, as both obstructive and nonobstructive lesions can contain calcific deposits in the vessel wall. However, increasing calcium scores are associated with an increasing likelihood of both obstructive 


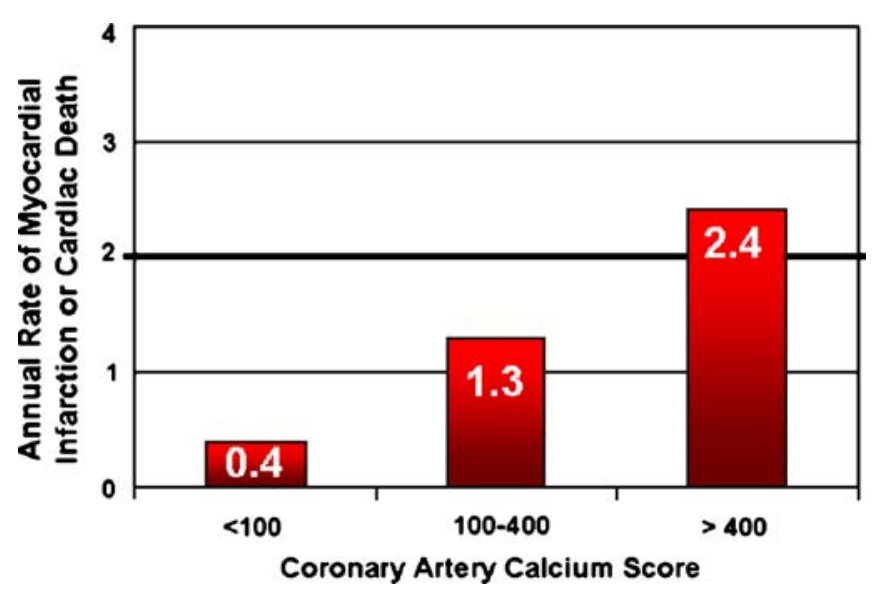

Fig. 2 Annual rate of myocardial infarction or cardiac death in categories of CAC burden in persons at intermediate risk based on convention risk factor assessment. In persons with a high CAC score $(>400)$, the annual event rate exceeds the threshold for intensive risk factor modification, i.e. $>2 \%$ per year (black line). A CAC score $>400$ in intermediate risk persons may therefore be considered as a risk equivalent (modified from [20])

disease and an increased severity (number of vessels involved) of CAD. Moderately high coronary calcium scores (approximately 150) in symptomatic patients are associated with a roughly $80 \%$ sensitivity and specificity for the presence of an obstructive coronary artery lesion (among patients referred to coronary angiography) [26, $28]$, an accuracy that is similar in magnitude to conventional stress tests $[38,39]$. Consistent with these data are findings from myocardial perfusion scintigraphy in which only $2 \%$ of patients with a CAC score $<100$ were shown to have a positive nuclear stress test [40]. A clinical application of these relationships has been demonstrated among emergency-department patients with chest pain, in whom a zero calcium score was associated with a very low risk of cardiovascular events [41]. Caution in the interpretation of zero calcium scores is warranted among individuals with a high pretest probability for CAD (e.g., young smokers) in whom false-negative studies may be observed [42].

In summary, the majority of expert writing committee members agreed that patients at low risk of CAD by virtue of atypical cardiac symptoms may benefit from CAC testing to help exclude the presence of obstructive CAD. CAC scoring may be a useful filter prior to invasive angiography or further stress testing [43, 44]. However, more data on direct comparisons with established forms of stress testing are needed. Currently, additional noninvasive testing in persons with a very high CAC score, e.g., $>400$, is not recommended as there is no evidence that such additional testing will improve appropriate selection of candidates for therapy. CAC CT was classified as "class IIb, level of evidence: B" when used to rule out obstructive CAD in patients with chest pain with equivocal or normal ECGs and negative cardiac enzymes, and in symptomatic patients in the setting of equivocal exercise stress tests [3].
Other clinical scenarios Serial imaging of CAC to assess disease progression is currently not indicated by the existing guidelines [3]; this issue is discussed later herein. Existing evidence on CAC CT has mostly been gathered from studies in Caucasian men, and caution is warranted in extrapolating existing data to other ethnic groups or women.

Implications for therapy

The NCEP/ATP III Guidelines have incorporated CAC CT as a complementary test to modify treatment intensity: "Measurement of coronary calcium is an option for advanced risk assessment in appropriately selected persons. In persons with multiple risk factors, high coronary calcium scores (e.g., $>75$ th percentile for age and sex) denote advanced coronary atherosclerosis and provide a rationale for intensified LDLlowering therapy. Moreover, measurement of coronary calcium is promising for older persons in whom the traditional risk factors lose some of their predictive power [34]. The use of CAC percentile ranks, as advocated in the NCEP guidelines, is especially important in young individuals whose absolute scores may be low, yet "high-for-age," indicating a high life-long risk, even though short-term risk over the next 5-10 years may be low. As risk-factor modification in high-risk subjects should be initiated as early as possible, such persons are likely appropriate candidates for intensive risk modification - a notion that needs to be further confirmed by prospectively collected outcome data.

\section{Limitations}

There is currently little evidence that CAC CT and knowledge of CAC score severity has an impact on the advice physicians give to patients or on patients' adherence to prescribed risk-factor modification efforts [45]. Further, CAC CT may improve risk stratification in selected populations, but currently the data are limited that CAC CT improves outcome. Accordingly, current evidence does not support lowering treatment intensity in intermediate risk subjects even if the CAC score is zero [4].

\section{Summary}

Current guidelines propose the use of CAC CT to improve risk stratification in subjects at intermediate 10-year risk of incident coronary events. The present writing committee agrees with this general recommendation.

\section{Race and calcium score}

There is still limited knowledge of the predictive value of CAC in non-Caucasians. It has been well documented that 
there is a notable difference in CAC accumulation not only between men and women, but also between subjects of different ethnicities and races. Doherty et al. [46] using subtraction fluoroscopy first noted a significantly lower prevalence of CAC in blacks than whites (35.5 vs. $59.9 \%$, $P=0.0001)$ and warned of the different prognostic significance of $\mathrm{CAC}$ in these races. Indeed, during a follow up of $70 \pm 13$ months, $23.7 \%$ of the black and $14.8 \%$ of the white screened population suffered an incident cardiovascular event (OR: 2.16, 95\% CI 1.34-3.48). The significant difference in prevalence and distribution of CAC assessed by CT in four races in the U.S. was recently confirmed by the Multi-Ethnic Study of Atherosclerosis (MESA). Bild et al. [16] showed that the prevalence of CAC on cardiac CT (score $>0$ ) was highest in whites followed by Chinese, Hispanics, and finally blacks. Santos et al. [47] showed that North American Caucasian subjects have more CAC than Caucasian subjects from Brazil and Portugal despite the higher prevalence of risk factors in the latter two ethnic groups. Interestingly, despite a substantial genetic similarity between Brazilian and Portuguese patients, and the presence of more smokers among the latter, Brazilians had a greater extent of CAC than Portuguese subjects. These findings mirrored the national mortality and morbidity statistics, indicating a greater cardiovascular event rate in the North American, followed by the Brazilian, and finally the Portuguese population.

Despite the noted differences in CAC scores, there is currently limited evidence of the prognostic significance of CAC in different races. Detrano et al. [48] showed that $\mathrm{CAC}$ is a strong predictor of cardiovascular death, nonfatal myocardial infarction, angina, and revascularization (total events=162) in all 6,722 MESA patients independent of race. Furthermore, $\mathrm{CAC}$ added incremental prognostic value beyond traditional risk factors for the prediction of events.

Recently, Nasir et al. [49] evaluated the use of CAC to predict all-cause mortality in 14,812 patients belonging to the same four races considered in MESA (505 deaths in 10 years of follow-up). Once again the prevalence of CAC was highest in whites, although blacks and Hispanics had a greater clustering of risk factors for CAD. Despite a lower prevalence of CAC and lower scores compared to the other races, black patients had the highest mortality rates even after multivariable adjustment for clinical risk factors and baseline CAC score $(P<0.0001)$. Compared with whites, the relative risk of death was 2.97 (CI:1.87-4.72) in blacks, 1.58 (CI: 0.92-2.71) in Hispanics, and 0.85 (CI: 0.47-1.54) in Chinese individuals. A 50-year-old black patient with a CAC score > 400 had an estimated loss of 7 years of life, as opposed to 2.5 years of life for a white patient with the same score.

Therefore, it would appear appropriate to consider CAC a good marker of risk in all races so far investigated, although the prognostic significance of score categories differs among racial groups. This underscores the importance of racially specific risk categories defined according to CAC score thresholds. An attempt at defining such categories was recently published by Sirineni et al. [50]. In their publication, the authors suggested substituting the chronological age of a patient undergoing CAC screening for his vascular age. The vascular age can be assessed according to the median CAC score for a subject of the same age, race, and sex. For example a 50-year-old black man with a CAC score of 40 should be considered 20 years older than his chronological age, since 40 is the median score of a 70 year-old black man in MESA. On the other hand, a score of 40 adds only 11 years of age to a 50 -year-old white man. The prognostic validity of this novel approach is still awaiting confirmation in prospective studies.

\section{The value of coronary artery calcium scoring in the elderly population}

The assessment of coronary calcification may have particular value in the elderly population. The potential for prevention of coronary heart disease (CHD) in older adults is large, since even a small reduction in risk-factor levels results in a considerable reduction in event rates. However, to identify asymptomatic elderly in the population at the highest risk of CHD is challenging. Office-based risk score algorithms such as the Framingham risk score [29] and the European SCORE [31] have an upper age threshold that limits their applicability to older adults. Furthermore, the predictive power of risk factors diminishes with increasing age [51-53]. Finally, age becomes the predominant factor in the algorithm in older adults, despite the fact that a fixed weight attributed to age does not take into account the individual variation in coronary plaque burden. On the basis of risk factors and age, the true CHD risk may be miscalculated, and this may lead to inaccurate selection of elderly patients for aggressive risk-factor modification.

CAC reflects the life-time impact of all atherosclerosis risk factors, both known and unknown, on the arterial wall [54]. Thus, this noninvasive measurement can provide a more accurate estimate of the accumulated plaque burden and CHD risk. So far, one population-based study has focused on the predictive value of CAC in the elderly: the Rotterdam Coronary Calcification Study (mean age: 71 years) [18]. During a mean follow-up period of 3.3 years, 50 of the 1,795 initially asymptomatic subjects had a coronary event. Increasing CAC score categories showed relative risks for CHD up to 8.2 (95\% CI: 3.3-20.5) for a CAC score above 1,000 , compared to zero or low CAC score $(0$ 100). Similar relative risks were found after adjustment for risk factors and in asymptomatic individuals over 70 years of age. Of interest, there was a very low probability of events in subjects with a low CAC score $(0-100)$. Furthermore, irrespective of the Framingham risk category (low-to-intermediate or high risk), increasing CAC score categories were strongly associated with the risk of events. Thus, a low CAC score in elderly may be as valuable a finding as in younger 
subjects. These results indicate that the CAC score is a very promising measurement to improve cardiovascular risk stratification in the elderly. In a recent publication, Abbott et al. [55] reported on 224 very old (age 84-96) Japanese men living in Hawaii followed for an average of 2.5 years after CAC imaging. A total of 17 deaths occurred during 2.5 years of follow-up and no death occurred in patients with a CAC score $<10$. As shown in the study by Vliegenthart et al. [18], the death rate increased significantly as the CAC score increased $(P<0.001)$. Finally, Newman et al. [56] measured CAC and carotid intima-media thickness in 559 patients (336 women) age 70-99 years. The top quartile of each measurement was associated with an approximately twofold increased risk of a combined cardiovascular disease endpoint.

Other population prospective studies have been conducted in a wide age range [20-22, 24, 36, 57]. Most of these studies did not specifically address the predictive value of CAC in older age. In a study by LaMonte et al. [22], CHD event rates adjusted for gender were presented in different age groups. In subjects over 65 years of age, a graded increase in event rates was seen for CAC scores $\geq 100$ and $\geq 400$ ( 7.1 and 8.2 per 1,000 person-years, respectively). Conversely, absence of CAC was associated with a very low event rate ( 0.9 per 1,000 person-years).

\section{Summary}

These data support the notion that CAC screening may be used in all age groups to adjust the relative risk level. They must, however, be considered preliminary; more research will be needed to demonstrate that expensive medical therapies can be withheld in the elderly with risk factors in the absence of CAC and to establish the best approach to managing older, asymptomatic patients with extensive CAC.

\section{Diabetes mellitus and coronary artery calcium}

Patients suffering from diabetes type-2 have been shown to harbor larger amounts of CAC than nondiabetic patients with the metabolic syndrome [58] and subjects of similar age and otherwise similar risk factor profile $[58,59]$. The extent of CAC in patients with type-2 diabetes is similar to that of patients with established CAD but without diabetes; diabetic women harbor as much CAC as diabetic men [60, 61]; and younger diabetic individuals have a plaque burden comparable to that of older nondiabetic individuals [62]. All of this confirms the clinical evidence that diabetes mellitus is associated with a very high prevalence of CAD; it negates the advantage of women over men and of youth over older age in prevalence and extent of atherosclerosis. Hoff et al. utilized a large database to calculate the age and gender normative (percentile) distribution of calcium scores in asymptomatic (self-reported) diabetic individuals [62].
Olson et al. [63] investigated the presence of CAC and prior CAD in 302 patients with diabetes mellitus type- 1 and a history of myocardial infarction, angina, or evidence of ischemia on stress testing or surface electrocardiograms. Among the subjects free of clinical CAD, $5 \%$ had a CAC score $\geq 400$ (large atherosclerosis burden), as compared to $25 \%$ of the subjects with prior angina or objective evidence of myocardial ischemia and $80 \%$ of the patients with myocardial infarction or luminal stenoses on invasive angiography. CAC showed a sensitivity of 84 and $71 \%$ for clinical CAD in men and women, respectively, and $100 \%$ sensitivity for myocardial infarction (MI) and obstructive CAD.

Limited data exist on outcome related to CAC in diabetic patients. Wong et al. [64] performed CAC screening and stress myocardial perfusion imaging (MPI) in 1,043 patients, 313 of whom were affected by either diabetes mellitus $(n=140)$ or the metabolic syndrome $(n=173)$. In patients with a CAC score $<100$, the prevalence of stressinduced MPI abnormalities was very low $(\sim 2 \%)$. However, in the presence of a metabolic disorder (diabetes mellitus or the metabolic syndrome), a CAC score between 100-399 or greater than 400 was associated with a greater incidence of ischemia than in patients without a metabolic disorder (13 vs. $3.6 \%, P<0.02$, and 23.4 vs $13.6 \%, P=0.03$, respectively). Similarly, Anand et al. [65] performed sequential CAC screening and MPI in 180 type- 2 diabetic patients. The incidence of myocardial ischemia was directly proportional to the CAC score. For type-2 diabetic patients with a CAC score of $0,11-100,101-400,401-1,000$, and $>1,000$, the incidence of myocardial ischemia on stress MPI was $0,18,23,48$, and $71 \%$, respectively. In summary, based on the Wong [64] and Anand data [65], type-2 diabetic patients with a CAC score $>100$ are expected to have an increased frequency of ischemia on MPI.

Two outcome studies addressed the question of whether CAC constitutes a risk for events in asymptomatic patients but came to opposite conclusions. The South Bay Heart Watch (SBHW) was a prospective cohort study designed to determine the relation between radiographically detectable $\mathrm{CAC}$ and cardiovascular outcome in high-risk asymptomatic adults [66]. Asymptomatic subjects $\geq 45$ years old with cardiac risk factors $(n=1,312)$ were recruited via massmailing advertisement in the Los Angeles area; of these $19 \%$ were diabetic patients. In a sub-analysis of the main database after a mean follow-up of 6 years, Qu et al. [66] found an increased risk of cardiovascular events (death, myocardial infarction, stroke, and revascularizations) in diabetic patients compared to nondiabetic subjects in the presence of CAC. However, the risk did not increase significantly as the CAC score increased. Raggi et al. [67] utilized a database of 10,377 asymptomatic individuals (903 diabetic patients), followed for an average of 5 years after CAC screening. The primary endpoint of the study was all-cause mortality. The authors showed that the risk of all-cause mortality was higher in diabetic patients than nondiabetic subjects for any degree of $\mathrm{CAC}$, and the risk 
increased as the score increased. Additionally, the absence of CAC predicted a low short-term risk of death $(\sim 1 \%$ at 5 years) for both diabetic patients and nondiabetic subjects [67]. Hence, both the presence and absence of CAC were important modifiers of risk even in the presence of established risk factors for atherosclerosis such as diabetes mellitus. This suggests that there is a great heterogeneity among diabetes mellitus patients and that risk stratification may be of benefit even in patients considered to be at highrisk of atherosclerosis complications.

\section{Summary}

The preceding discussion suggests that CAC imaging techniques may be very helpful to the practicing physician faced with the dilemma of accurate risk assessment even in diabetic patients at high risk. However, as is the case with other subsets of patients, further research will be needed to confirm the prognostic role of CAC in diabetes mellitus.

\section{Renal failure and coronary artery calcium}

Both EBCT and MSCT have been utilized in the recent past to investigate the natural history and pathogenesis of CAC, as well as the impact of different therapeutic strategies in chronic kidney disease (CKD). Evidence indicates that as the estimated glomerular filtration rate (eGFR) declines, the prevalence of CAC increases. In fact, the prevalence of CAC was reported to be $40 \%$ in 85 predialysis patients as opposed to $13 \%$ in controls with normal renal function [68]. In a prospective study of 313 high-risk hypertensive patients, a reduced eGFR was shown to be the major determinant of the rate of progression of CAC (OR for calcium progression in the group with eGFR $<60 \mathrm{ml} / \mathrm{min}$ : 2.1; 95\% CI 1.2-3.7) [69]. Consistent with these findings, Sigrist et al. [70] reported a prevalence of CAC of $46 \%$ in 46 pre-dialysis patients compared to 70 and $73 \%$, respectively, in 60 hemodialysis and 28 peritoneal dialysis patients $(P=$ $0.02)$. Hence, it appears that the prevalence of CAC increases with declining renal function and after initiation of dialysis. Of note, CAC was reported in $\sim 60 \%$ of patients new to hemodialysis [71] and in as many as $80-85 \%$ of adult prevalent hemodialysis patients [72] in two prospective, randomized studies. In a small longitudinal study, the baseline CAC score measured by EBT in 49 prevalent hemodialysis patients was on average two- to fivefold higher than in age- and sex-matched individuals with established CAD. A repeat $\mathrm{CT}$ after an interval of 12 months showed significant progression of CAC $(P<0.05)$ [73].

A number of factors have been associated with progression of CAC in dialysis patients. Associations with age and duration of dialysis [72, 74], diabetes mellitus [72], abnormalities of mineral metabolism [75-77] as well as use and dose of calcium-based phosphate binders $[78,79]$ have all been reported. To investigate the impact of therapy for hyperphosphatemia on the progression of CAC, a randomized clinical trial compared the effect of Sevelamer (Genzyme, Cambridge, MA, USA), a nonabsorbable polymer with gut phosphate-binding ability, and calcium-based phosphate binders in 200 hemodialysis patients for 1 year [78]. Throughout the study, both drugs provided a comparable phosphate control (mean phosphate $=5.1 \mathrm{mg}$ / $\mathrm{dl}$ ), although a significantly higher serum calcium concentration $(P=0.002)$ was noted in the calcium-salts-treated arm. At study completion, Sevelamer-treated subjects were less likely to have experienced CAC progression (median absolute progression of CAC score 0 vs $36.6, P=0.03$ and aorta 0 vs $75.1, P=0.01$, respectively) [78].

In a smaller series of 129 patients new to hemodialysis [80], subjects treated with calcium-containing phosphate binders showed a more rapid and more severe increase in CAC score compared with those receiving Sevelamer $(P=$ 0.056 at 12 months, $P=0.01$ at 18 months) [80]. In the same series, all-cause mortality was strongly associated with the baseline CAC score and was significantly lower in the Sevelamer arm after 4.5 years of follow-up $(P=0.02)$ [71]. Even more surprisingly, mortality was extremely low (3.9\%/year) in patients with zero calcium score. This stands in contrast with a reported mortality of $\sim 20-25 \% /$ year in patients undergoing hemodialysis. CAC scores were also shown to be predictive of an unfavorable outcome in dialysis patients by Matsuoka et al. [81]. The authors followed 104 chronic hemodialysis patients for an average of 43 months after a screening EBT. Patients were divided into two groups according to a baseline CAC score falling below or above the median for the group (score $=200$ ). The 5 -year cumulative survival was significantly lower for patients with a CAC score $>200$ than for those with a score $<200$ (67.9 vs. $84.2 \%, P=0,0003$ ).

\section{Summary}

CAC appears to be predictive of an adverse outcome in CKD patients and its absence has been linked with a very low event rate.

\section{The value of the zero calcium score-asymptomatic patients}

The presence of coronary calcification is, especially with advancing age, a sensitive but unspecific finding. As discussed above, many studies have emphasized the graded increase in CHD risk with increasing calcium scores. However, an even more clinically relevant finding may be the absence of CAC. In a large population of over 10,000 individuals screened for CAC, all-cause mortality was assessed during a 5-year follow-up period. With a zero or very low $(<10)$ calcium score, the investigators reported a 
very low probability of mortality, $\sim 1.0 \%$ at the end of followup [82]. This finding was confirmed in a study by Budoff in 25,253 individuals, in which only $0.4 \%$ of the individuals with a negative calcium score died during almost 7 years of follow-up, compared to $3.3 \%$ of individuals with a positive CAC score [57]. In prospective studies in which CHD was used as outcome measure, a zero or very low calcium score was associated with a very low probability of events during follow-up [21, 23, 24, 36, 83]. Church et al. reported a relative risk of coronary events in subjects without CAC compared to those with a positive calcium score of 0.13 (95\% CI, 0.06 0.30) [83]. Cumulative incidences in studies with a follow-up period of 3-5 years ranged between 0.1 and $0.7 \%$ (Table 2). One study showed a somewhat higher cumulative incidence of $4.4 \%$ during more than 6 years [20]. This may be partly explained by the different $\mathrm{CT}$ protocol (6-mm slicing), which may have resulted in missing calcified lesions.

Four studies have specifically compared the prognosis for men and women in the absence of CAC. Raggi et al. found no difference in all-cause mortality after 5 years of follow-up in over 4,000 women and over 6,000 men with a very low CAC score $(<10): 1.6$ vs $1.5 \%$ [84]. Recently, the results from three studies in which CHD was the outcome [21-23] were used in a meta-analysis [85]. In total, the analysis included 3,862 women and 5,548 men with absent or minimal CAC. The annual CHD event rate was very similar in women and men: 0.2 vs. $0.3 \%$. When only women and men with no CAC were studied, rates were somewhat lower $(0.16$ vs. $0.27 \%)$ but again not significantly different. Thus, absent or very low CAC score carries the same prognostic value in both genders.

Interestingly, even in the presence of cardiovascular risk factors, the negative predictive value of absent or minimal CAC appears to be very high. In the aforementioned study in which all-cause mortality was the outcome [82], further investigations were performed according to smoking status and diabetes status of the participants [42,67]. Absence of CAC was noted in about $30 \%$ of individuals with diabetes, and in $50 \%$ of smokers. Little or no CAC was associated with a near $100 \%$ survival in nonsmokers as well as smokers, and nondiabetic as well as diabetic subjects.

As discussed in the previous section, Block et al. [71] reported a very low mortality rate for hemodialysis patients

Table 2 Zero or very low calcium score and events in asymptomatic populations

\begin{tabular}{|c|c|c|c|c|c|c|c|c|c|}
\hline Author & Year & $\begin{array}{l}\text { Total } \\
(n)\end{array}$ & $\begin{array}{l}\text { Subgroups } \\
(n)\end{array}$ & $\begin{array}{l}\text { Mean } \\
\text { age } \pm \mathrm{SD} \\
\text { (years) }\end{array}$ & $\begin{array}{l}\text { Lowest } \\
\text { calcium score } \\
\text { category }\end{array}$ & $\begin{array}{l}\text { Percentage } \\
\text { in lowest } \\
\text { category }\end{array}$ & $\begin{array}{l}\text { Mean } \\
\text { follow-up } \pm \\
\text { SD (years) }\end{array}$ & Outcome & $\begin{array}{l}\text { Cumulative } \\
\text { incidence } \\
(\%)\end{array}$ \\
\hline Raggi [24] & 2000 & 632 & & $52 \pm 9$ & 0 & 46 & $2.7 \pm 0.6$ & $\mathrm{MI} /$ cardiac death & 0.3 \\
\hline Shaw $[82]^{\mathrm{a}}$ & 2003 & 10,377 & & $53 \pm 10$ & $0-10$ & 57 & $5.0 \pm 3.5$ & All-cause mortality & 1.0 \\
\hline Raggi $[84]^{\mathrm{a}}$ & 2004 & 10,377 & $\begin{array}{l}\text { 4,191 Women } \\
6,186 \text { Men }\end{array}$ & $\begin{array}{l}55 \pm 11 \\
52 \pm 11\end{array}$ & $0-10$ & $\begin{array}{l}68 \\
50\end{array}$ & $5.0 \pm 3.5$ & All-cause mortality & $\begin{array}{l}1.6 \\
1.5\end{array}$ \\
\hline Raggi [67] ${ }^{\mathrm{a}}$ & 2004 & 10,377 & $\begin{array}{l}903 \text { Diabetics } \\
9,474 \\
\text { Nondiabetics }\end{array}$ & $\begin{array}{l}57 \pm 10 \\
53 \pm 10\end{array}$ & 0 & $\begin{array}{l}30 \\
51\end{array}$ & $5.0 \pm 3.5$ & All-cause mortality & $\begin{array}{l}1.2 \\
0.6\end{array}$ \\
\hline Shaw $[42]^{\mathrm{a}}$ & 2006 & 10,377 & $\begin{array}{l}\text { 4,113 Smokers } \\
6,264 \\
\text { Nonsmokers }\end{array}$ & $\begin{array}{l}53 \pm 10 \\
54 \pm 11\end{array}$ & $0-10$ & $\begin{array}{l}\text { About } 49 \\
\text { About } 63\end{array}$ & $5.0 \pm 3.5$ & All-cause mortality & $\begin{array}{l}0.5 \\
0.3\end{array}$ \\
\hline Kondos [21] & 2003 & 5,635 & $\begin{array}{l}\text { 1,484 Women } \\
\text { 4,151 Men }\end{array}$ & $\begin{array}{l}54 \pm 9 \\
50 \pm 9\end{array}$ & 0 & $\begin{array}{l}49 \\
26\end{array}$ & $3.0 \pm 1.0$ & MI/CHD death & $\begin{array}{l}0.3 \\
0.3\end{array}$ \\
\hline Greenland [20] & 2004 & 1,029 & & $66 \pm 8$ & 0 & 31 & $6.3 \pm 1.5$ & MI/CHD death & 4.4 \\
\hline Arad [36] & 2005 & 4,613 & & $59 \pm 6$ & 0 & 33 & 4.3 & $\begin{array}{l}\mathrm{MI} / \mathrm{CHD} \text { death/ } \\
\text { revascularizations }\end{array}$ & 0.5 \\
\hline Vliegenthart [18] & 2005 & 1,795 & & $71 \pm 6$ & $0-100$ & 50 & $3.3 \pm 0.8$ & MI/CHD death & 0.7 \\
\hline Taylor [23] & 2005 & 1,983 & $\begin{array}{l}356 \text { Women } \\
1,627 \text { Men }\end{array}$ & $43 \pm 3$ & 0 & $\begin{array}{l}92 \\
78\end{array}$ & $3.0 \pm 1.4$ & $\begin{array}{l}\text { MI/CHD death/ } \\
\text { unstable angina }\end{array}$ & $\begin{array}{l}0.0 \\
0.2\end{array}$ \\
\hline Church $[83]^{\mathrm{b}}$ & 2007 & 10,746 & & $54 \pm 10$ & 0 & 53 & $3.5 \pm 1.4$ & MI/CHD death & 0.1 \\
\hline LaMonte $[22]^{\mathrm{b}}$ & 2005 & 10,746 & $\begin{array}{l}\text { 3,911 Women } \\
\text { 6,835 Men }\end{array}$ & $\begin{array}{l}54 \pm 10 \\
53 \pm 10\end{array}$ & 0 & $\begin{array}{l}71 \\
39\end{array}$ & $3.5 \pm 1.4$ & MI/CHD death & $\begin{array}{l}0.1 \\
0.1\end{array}$ \\
\hline Budoff [57] & 2007 & 25,253 & & $56 \pm 11$ & 0 & 44 & $6.8 \pm 3.0$ & all cause mortality & 0.4 \\
\hline
\end{tabular}

$C H D$ Coronary heart disease, $M I$ myocardial infarction, $S D$ standard deviation Cumulative incidence derived from published data or calculated

${ }^{a}$ Same study, analysis in different subgroups

${ }^{\mathrm{b}}$ Same study, analysis in different subgroups 
without evidence of CAC (3.9\%/year); this is in contrast with the extremely high mortality rate $(\sim 25-30 \% /$ yearly) typically quoted for this category of patients. Thus, the absence of CAC may be an important modifier of the risk of events even in the presence of cardiovascular risk factors. The high negative predictive value of a zero CAC score is extremely valuable, considering that a large number of asymptomatic individuals have no CAC. In various studies, absence of CAC was noted in $26-92 \%$ of individuals, depending on the age of the individuals. Hence, a zero CAC score may have important implications in daily clinical practice and at a population level. The most important question from a population and societal point of view is whether individuals without CAC should be considered at low risk, even in the presence of cardiovascular risk factors, and therefore be spared therapies such as aspirin and cholesterol-lowering medications. Although the current evidence is substantial, such a notion cannot be endorsed at this time in the absence of prospective, randomized trials.

\section{The value of zero calcium score-symptomatic patients}

Calcium score and prediction of obstructive coronary artery disease on angiography

As outlined above, a negative CAC score has a high negative predictive value in asymptomatic patients of both genders and even in patients with risk factors such as smoking, diabetes, or renal failure. In symptomatic patients where CAD is suspected, can a zero or a minimal CAC score (e.g., $<10$ ) be used as a filter to rule out obstructive CAD? Several investigators have addressed this point. Becker et al. studied 1,347 symptomatic subjects with suspected CAD [86]. Sensitivity, specificity, and predictive accuracy were calculated for different calcium thresholds for prediction of CAD. In $720(53 \%)$ subjects, invasive angiography revealed a lumen diameter stenosis greater than $50 \%$. Patients with obstructive CAD had significantly higher total calcium scores than patients without $\operatorname{CAD}(P=0.001)$. The overall sensitivity of any CAC score to predict stenosis was $99 \%$, with a specificity of $32 \%$. An absolute score cutoff $\geq 100$ and an age- and sex-specific score $>75$ th percentile were identified as the cutoff levels with the highest sensitivities (86-89\%) and lowest false-positive rates (20-22\%). Absence of CAC was highly accurate for exclusion of CAD in subjects older than 50 years (negative predictive value $=$ 98\%). The authors concluded that the presence of CAC on MDCT in symptomatic patients is accurate for prediction of obstructive CAD and that its absence is associated with a high negative predictive value for exclusion of CAD.

Several other studies investigated the presence of noncalcified plaques and obstructive lesions in patients with a low or zero CAC score. Cheng et al. assessed the presence and severity of noncalcified coronary plaques on 64-MDCT coronary angiography in 554 symptomatic patients with low to intermediate pretest likelihood for CAD and zero or low CAC score (low score: men, score $<50$; women, score <10) [87]. The authors intended to elucidate how well absence of CAC predicts the absence of obstructive noncalcified coronary artery plaque (NCAP). Compared with patients with absent CAC, those with a low CAC score had markedly increased rates of critical luminal stenoses $(8.7$ vs. $0.5 \%, P<0.001)$. The authors concluded that in symptomatic patients with low to intermediate pretest probability of CAD, absence of CAC predicts very low prevalence of occlusive NCAP. Nonetheless, low but detectable CAC scores were significantly less reliable in excluding the presence of plaque that at times could be obstructive.

Leschka et al. recently studied the potential of using the CAC score to improve the diagnostic accuracy of MDCT angiography [88]. They evaluated 74 consecutive patients who underwent CAC scoring, MDCT angiography, and invasive angiography. Segments that were not evaluable on MDCT angiography were considered to be false-positive. When using CAC scores of 0 to exclude stenoses and $\geq 400$ to predict stenosis for segments with nonevaluative segments, the per-patient sensitivity and specificity improved from 98 and $87 \%$ to 98 and $100 \%$, respectively. Only the zero CAC score was found to be helpful to exclude stenoses as a high CAC score often corresponds to more than one stenosis in the coronary artery tree.

In a study by Rubinshtein et al. [89], the severity of CAD was examined using 64-MDCT angiography in patients who underwent testing due to chest pain syndromes and had a zero or low CAC score. Of 668 consecutive patients, 231 had a low score $(<100)$ or absent CAC. Obstructive CAD was present in 9 of 125 patients $(7 \%)$ with a 0 CAC score, and in 18 of $106(17 \%)$ with a low score (CAC: 1-100).

\section{Summary}

In conclusion, absent CAC seems to be an excellent filter for exclusion of obstructive CAD in symptomatic patients with intermediate to high pretest likelihood of obstructive CAD. A low CAC score, however, is more controversial as a number of studies showed that the presence of noncalcified and potentially obstructive lesions is higher in patients with low CAC scores and symptoms compared to patients with a score of zero.

The value of zero calcium score to rule out coronary artery disease in symptomatic patients: comparison to treadmill stress testing and nuclear stress tests

In discussing the potential value of a zero CAC score in symptomatic patients for a reliable exclusion of CAD, other noninvasive tests such as ECG stress testing or nuclear stress testing have to be considered. Exercise stress 
testing is often used as the initial noninvasive diagnostic test in symptomatic patients with suspected obstructive CAD. Positive standard ECG criteria are quite specific for obstructive CAD, but there may be a substantial number of false-negative tests, including patients with severe disease. Also, exercise stress tests frequently yield equivocal results. Lamont et al. assessed the value of combining CAC screening with a stress test to reduce the high falsepositive rate seen with treadmill stress test (TMST) alone [90]. A CAC score was obtained by EBT in 153 symptomatic patients who underwent coronary angiography because of a positive TMST. The TMST false-positive rate was $27 \%$ ( 41 of 153 ). In these patients, a CAC score of zero resulted in a negative predictive value of $93 \%$. The authors concluded that the absence of CAC reliably identified patients with a false-positive TMST result. Raggi et al. [44] showed that in symptomatic patients with low to intermediate pretest probability of disease (5$50 \%$ ), a CAC score of zero can be reliably used to exclude obstructive $\mathrm{CAD}$ and that calcium scoring as the initial test to investigate presence of CAD provides a substantial cost benefit over a pathway based on exercise stress testing.

Berman et al. [40] described the relationship between stress-induced myocardial ischemia on single-photon emission computed tomography (SPECT) perfusion studies and CAC. Including a total of 1,195 patients without known $\mathrm{CAD}, 51 \%$ asymptomatic, the frequency of ischemia by SPECT was compared to the magnitude of CAC. The frequency of ischemic SPECT was $<2 \%$ with CAC scores $<100$ and increased progressively for CAC $>100(P$ for trend $<0.0001)$. Patients with symptoms and CAC scores $>400$ had higher likelihood of myocardial ischemia versus those without symptoms $(P<0.025)$. The authors concluded that ischemic SPECT is associated with a high likelihood of subclinical atherosclerosis by CAC, but it is rarely seen for $\mathrm{CAC}$ scores $<100$. In most patients, low CAC scores appear to obviate the need for subsequent noninvasive testing. Patients with normal perfusion studies, however, frequently had extensive nonobstructive atherosclerosis by CAC criteria.

Geluk et al. determined the efficiency of a screening protocol based on CAC scores compared with exercise testing in patients with suspected CAD, a normal ECG, and troponin levels [43]. Three hundred and four patients were enrolled in a screening protocol that included CAC scoring by EBT and exercise testing. Decision-making was based on CAC scores. When the CAC score was $\geq 400$, coronary angiography was recommended. When the $\mathrm{CAC}$ was $<10$, patients were discharged. Exercise tests were graded as positive, negative, or nondiagnostic. The combined endpoint was defined as coronary event or obstructive CAD at coronary angiography. During $12 \pm 4$ months, CAC $\geq 400$, 10-399, and $<10$ were found in 42,103 , and 159 patients and the combined endpoint occurred in $24(57 \%), 14$ $(14 \%)$, and 0 patients $(0 \%)$, respectively. In 22 patients (7\%), myocardial perfusion scintigraphy was performed instead of exercise testing due to the inability to perform an exercise test. A positive, nondiagnostic, and negative exercise test result was found in 37,76 , and 191 patients, and the combined endpoint occurred in $11(30 \%), 15$ $(20 \%)$, and 12 patients (6\%), respectively. Receiveroperator characteristics curves showed that the area under the curve of 0.89 (95\% CI: $0.85-0.93)$ for CAC was superior to 0.69 (95\% CI: $0.61-0.78)$ for exercise testing $(P<0.0001)$. The authors concluded that measurement of CAC is an appropriate initial screening test in a welldefined low-risk population with suspected CAD.

The value of zero calcium score in patients presenting with acute chest pain to the emergency department

The use of CAC assessment was briefly discussed in a recent consensus paper on the use of MDCT for acute chest pain $[91,92]$. The use of CAC screening has been described in patients with angina-like symptoms and negative cardiac enzymes presenting to the emergency department (ED). Laudon et al. performed CAC scoring in the emergency department in 104 patients and noted a negative predictive value for CAD of $100 \%$ for a CAC score of zero [93]. McLaughlin et al. reported a negative predictive value of $98 \%$ in 134 patients in a similar ED setting [94]. Georgiou et al. followed 198 patients presenting to the ED with chest pain and normal ECG and cardiac enzymes and found that the presence of any $\mathrm{CAC}$ is a strong predictor for future cardiac events. Conversely, patients without CAC may safely be discharged from the ED given the extremely low rate of future events ( 0.1\%/year) [41]. Nonetheless, after reviewing the available evidence, Andrews concluded that currently existing data do not sufficiently support the widespread use of CAC CT in patients with acute chest-pain syndromes [95]. Even so, in patients at low pretest likelihood of CAD presenting with angina-like symptoms to the ED, a negative CAC score can possibly be used to rule out an acute coronary syndrome. In conclusion, the available single-center studies based on a limited number of patients indicate that the negative predictive value of a zero CAC is high $(>90 \%)$. However, the positive predictive value is somewhat lower, rendering CAC screening a highly sensitive, but poorly specific modality for the diagnosis of acute coronary syndromes.

\section{Calcium score progression: interpretation}

Serial changes in CAC score may have important implications for monitoring the response of atherosclerotic disease to the initiation of or changes in plaque-altering medical therapy as well as for identifying patients with more aggressive disease who are at high risk for incident CAD [4]. In this section, we will discuss the methodological approaches to calculating 
CAC progression as well as provide a synopsis of the available literature on the utility of sequential CT imaging to evaluate atherosclerotic disease progression.

\section{Serial testing paradigm}

Serial testing is based on the concept that changes in CAC are valid markers of varying atherosclerotic disease states [96]. Furthermore, a change in CAC may serve as a surrogate for clinical outcomes or disease activity and, as such, provide clinically useful information to guide further patient management [97-102]. The paradigm of using imaging as a surrogate outcome has been advanced in the oncologic PET literature [103]. The Response Evaluation Criteria in Solid Tumors (RECIST) provide definable criteria for partial or complete response to therapies of target and nontarget lesions.

Using this type of sequential monitoring, a positive change in CAC score above a given threshold signifies progressive disease, minimal or no changes in CAC score identify patients with stable disease, and a reduction in CAC score beyond a given limit defines patients exhibiting regression in their underlying disease. With regards to the latter, it is still very controversial whether CAC truly regresses. As such, this document will focus on defining rapidly and slowly progressive disease states.

\section{Reproducibility of CAC CT and its determinants}

A major consideration for interpretation of changes in CAC between serial CT examinations is the variability of repeat imaging. Interexamination variability is affected by image artifacts including motion, noise, and partial volume averaging that are highly dependent on the specific imaging protocol as well as the extent of CAC burden. Optimal timing of ECG triggering can reduce variability of Agatston scores from $>30$ to $<15 \%$ with EBT [104-106]. The correlation coefficients across CAC measurements, including Agatston score (AU), calcium volume score (CVS), or MS, are excellent $(R \geq 0.96, n=161)[11,107]$. CVSs improve reproducibility only marginally compared to Agatston scores. The square root of the CVS has, however, been suggested to reduce interexamination variability [108].

Differences between types of CT systems are very small after adjustment for body mass index and CAC burden [10]. In the MESA study, mean relative differences between CT examinations at different times were $20.1 \%$ for the Agatston score and $18.3 \%$ for the interpolated CVS $(P<0.01)[10]$, which are in line with previous reports. Of note, these data were obtained from CT performed at $80 \%$ of the RR interval, which is associated with a lower reproducibility as compared to earlier triggering.

Data acquired with 4-slice CT systems were reported to have higher rates of misregistration compared with EBT [10].
Motion artifacts were also higher in these CT systems compared to EBT machines, while image noise was lower [10]. The reproducibility of the calcium score has improved with the introduction of 16-slice and more recently 64-slice MDCT systems. The variability is best with thinner slices, higher calcium scores and with retrospective acquisition mode, although this is associated with a higher radiation dose for the patient. Currently, the reported variability of the Agatston, volume, and mass score on 16- to 64-slice MDCT ranges between 8 and $18 \%$ (lower end of the range with 64-slice MDCT) on sequential examinations performed within minutes of each other [109-111]. Given the radiation exposure, especially with MDCT systems, the benefit-risk ratio and time intervals of repeat CT must be considered individually, especially when women and young men are examined.

Clinical thresholds of coronary artery calcium progression

Progression of CAC is generally calculated as a percent or absolute change from the baseline score using either the Agatston score, CVS, or MS [99, 104, 112-119]. Raggi et al. defined a change $>15 \%$ as true progression [98], while Hokanson et al. suggested a CAC progression $\geq 2.5 \mathrm{~mm}^{3}$ of the square root of the initial volume score as a useful threshold of progression [108].

The absolute change in CAC is expected to be greater in patients with a higher baseline score (Figs. 3 and 4) [98, $115,116]$, although the absolute differences reflect minor changes compared to baseline. Larger percent score changes are expected in patients with a low index CAC score (e.g., index CAC score of 10 to repeat score of $20=$ progression of $100 \%$ ) and do not necessarily reflect a clinically relevant change.

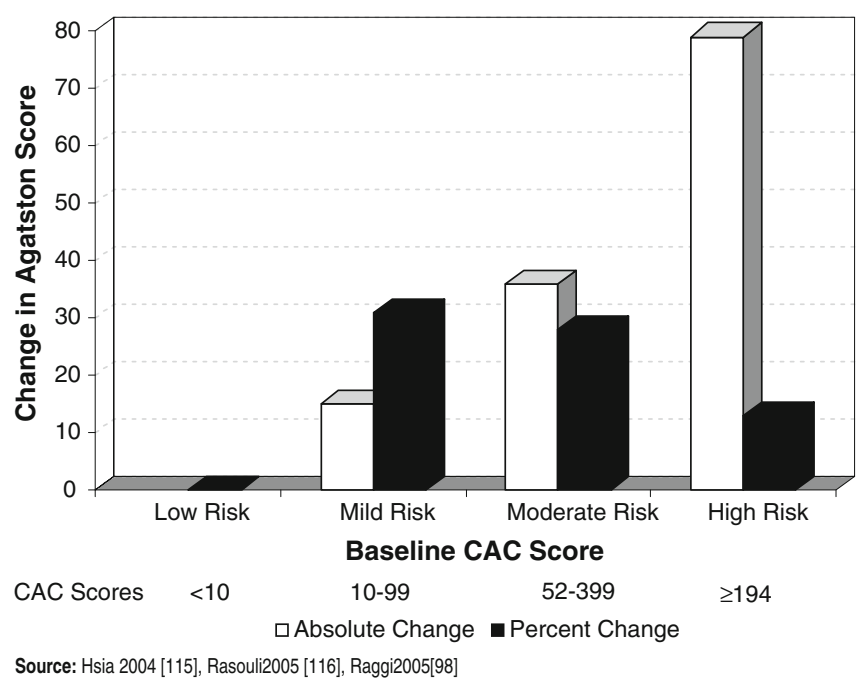

Fig. 3 The absolute and percent change in baseline Agatston score on serial $\mathrm{CT}$ imaging 


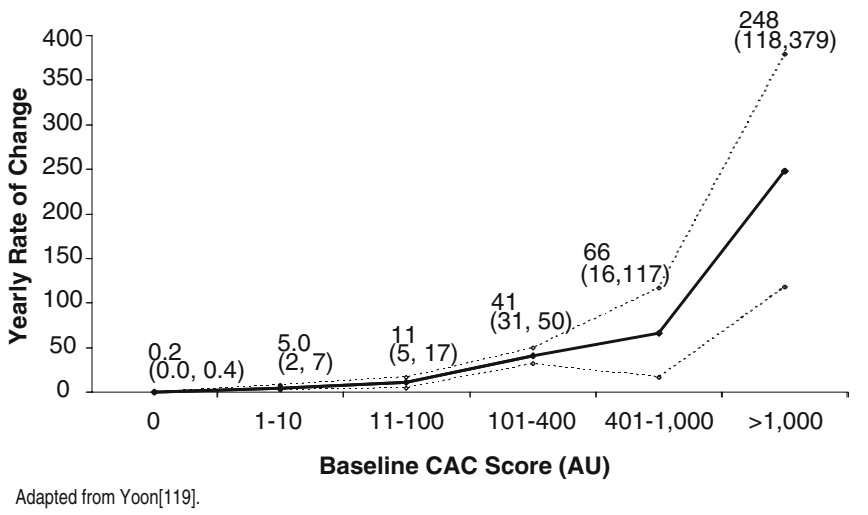

Fig. 4 Expected yearly rate of change (95\% confidence intervals) from baseline for coronary artery calcium scores ranging from 0 to $\geq 1,000$ Agatston units (AU)

Clinical interpretation of changes in coronary artery calcium

For most patients in the various risk groups in Fig. 5, the error in score reproducibility would not affect their clinical management, unless scores are close to adjacent risk groups. Variability increases with CAC score and may be as much as $200-380$ units for scores of 400 or higher (Fig. 5) [120]. As scores of 100 or 400 may trigger more aggressive postscreening management or follow-up ischemia testing, clinicians should rely less on the absolute thresholds and more on a combination of CAC score with the patient's clinical presentation and cardiac risk factor profile. Aggressive management is indicated for scores of 1,000 or higher (very high risk CAC), and it is unlikely that the expected variability about this point estimate will change clinical care [99, 104, 112-119].

Rates of coronary artery calcium progression and its determinants

In subjects at average Framingham risk, the annual CAC progression rates typically range from 20 to $24 \%$ per year using either the Agatston or the CVS [99, 104, 112-119]. Factors that may significantly modify rate of change include the patient's baseline CAC score, gender, age, family history of premature $\mathrm{CAD}$, ethnicity, diabetes and glycemic control, body mass index, hypertension, and renal insufficiency [98, 121-125]. Further, the longer the interval from baseline to repeat $\mathrm{CAC} \mathrm{CT}$, the greater the expected change [117]. However, the absolute change will be greater but the relative change may be smaller. The score does not continue to grow exponentially and the rate of growth eventually tapers off. Most patients exhibit a positive change in CAC scores over time [99, 112-114, 116, 118] although some patients (29-34\%) exhibit no change if they are at low Framingham risk, including women, or have a baseline score of zero (38\%) [114]. In patients with an initial zero score, a repeat $\mathrm{CT}<5$ years after the initial examination may not be useful for clinical purposes [114].

Results of randomized clinical trials on effect of statin therapy on coronary artery calcium progression

A number of observational studies (Table 3) and randomized clinical trials (Fig. 6) have evaluated change in CAC following treatment with statin therapy. In four observational reports, untreated patients had an average CAC score progression of $36 \%$ [98, 126-128]. By comparison, statin therapy attenuated changes in CAC scores averaging 13\% (Table 3) [98, 126-128].

However, these promising observational data were contradicted by large randomized clinical trials showing similar changes in CAC scores following placebo and/or moderate-intensive statin therapy (Fig. 6). Except for a preliminary pilot trial [129], all other randomized trials have failed to confirm the preliminary observational findings (Fig. 6). Comparison of intensive vs. moderate statin therapy showed no difference in CAC progression (Fig. 6) $[100,130]$. The lack of an effect in these clinical trials suggests that a longer observational time period may be warranted and that statins may reduce cardiac events independent of an effect on calcified plaque [4]. Further, these trials often did not consider or plan management of other CV risk factors that may confound the lack of therapeutic benefit [4]. There are ongoing trials using CAC as a surrogate where additional evidence may be put forth on the benefit of serial imaging $[131,132]$. Finally, other treatments have been tested as far as an effect on CAC progression. In the Women's Health Initiative (WHI), menopausal women between the ages of 50-59 years were randomized to treatment with conjugated estrogens or placebo [133]. In a substudy of the WHI, 1,064 women were submitted to CAC screening after 8.7 years from trial initiation. Women receiving estrogens showed a lower CAC score compared with those receiving placebo $(83.1$ vs. $123.1, P=0.02$ )

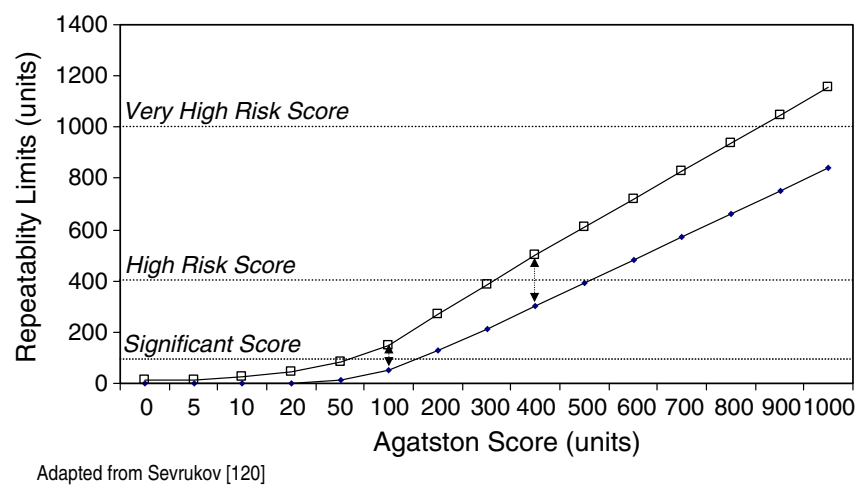

Fig. $595 \%$ Confidence intervals for repeatability of coronary artery calcium scores from 0 to $\geq 1,000$ 
Table 3 Percent yearly progression from observational cohorts of consecutive patient series, with average Framingham risk, and evidence of coronary artery calcium (CAC) on baseline CT

\begin{tabular}{|c|c|c|c|c|c|c|}
\hline Author & Year & Number & Entry criteria & Score & $\begin{array}{l}\text { Testing period } \\
\text { (years) }\end{array}$ & $\begin{array}{l}\text { Percent change in } \\
\text { CAC score/year }\end{array}$ \\
\hline Budoff [126] & 2000 & 299 & Consecutive patients & $\mathrm{AU}$ & $\geq 1$ & 33 \\
\hline Shemesh [117] & 2001 & 116 & Asymptomatic hypertensive patients & AU & $1,2,3$ & $\begin{array}{l}\text { Year 1: } 18 \\
\text { Year 2: } 31 \\
\text { Year 3: } 41\end{array}$ \\
\hline Sutton-Tyrrell [118] & 2001 & 80 & Middle-aged women & $\mathrm{AU}$ & 1.5 & 11 \\
\hline Yoon [119] & 2002 & 217 & Consecutive subjects & $\begin{array}{l}\text { AU } \\
\text { CVS }\end{array}$ & 2.1 & $\begin{array}{l}34 \\
29\end{array}$ \\
\hline Raggi [99] & 2003 & 772 & Consecutive patients & CVS & 2.2 & 26 \\
\hline Hsia [115] & 2004 & 94 & Healthy postmenopausal women with $\mathrm{CAC} \geq 10$ & AU & 3.3 & 27 \\
\hline Budoff [113] & 2005 & 177 & Postmenopausal patients & $\mathrm{AU}$ & $\geq 1$ & $15-22$ \\
\hline Rasouli [116] & 2005 & 133 & Asymptomatic patients & $\mathrm{AU}$ & 1.7 & $17-22$ \\
\hline Gopal [114] & 2006 & 710 & Consecutive patients $\mathrm{w} / \mathrm{CAC}=0$ & AU & $\geq 1$ & $\begin{array}{l}\text { Mean } \pm \text { SD: } 1 \pm 3 \\
\text { Median (IQR): } 0(0-0.8)\end{array}$ \\
\hline Becker [112] & 2007 & 277 & Postmenopausal women & CVS & 3.3 & 18 \\
\hline Summary data & & 2,875 & & $\begin{array}{l}\text { AU } \\
\text { CVS }\end{array}$ & & $\begin{array}{l}20 \\
24\end{array}$ \\
\hline
\end{tabular}

$C A C$ Coronary artery calcium, AU Agatston units, CVS calcium volume score

${ }^{a}$ Or mean \pm standard deviation (SD) or median with interquartile range (IQR) in the Gopal series

\section{Cardiovascular prognosis related to coronary artery calcium progression}

Despite the lack of an effect of statins on CAC progression, several reports have noted that a rapid change in CAC score is associated with worse clinical outcomes including incident MI [97, 99]. In one report of 495 patients, subjects who experienced an acute MI experienced greater degrees of CAC progression compared to event-free survivors (42 \pm $23 \%$ vs. $17 \pm 25 \%, P<0.0001)$ [97]. Patients with and without

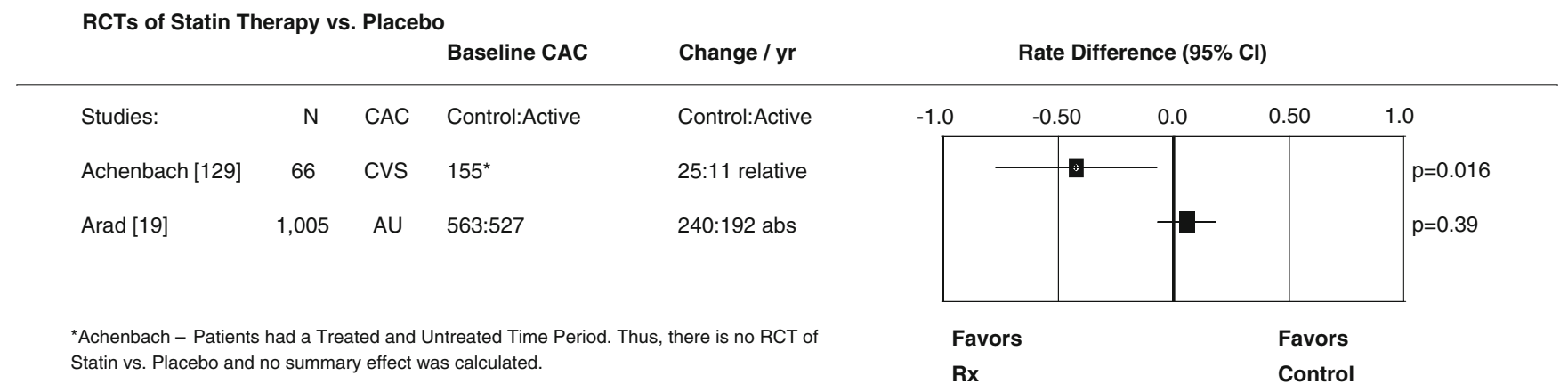

RCTs of Moderate vs. Intensive Statin Therapy

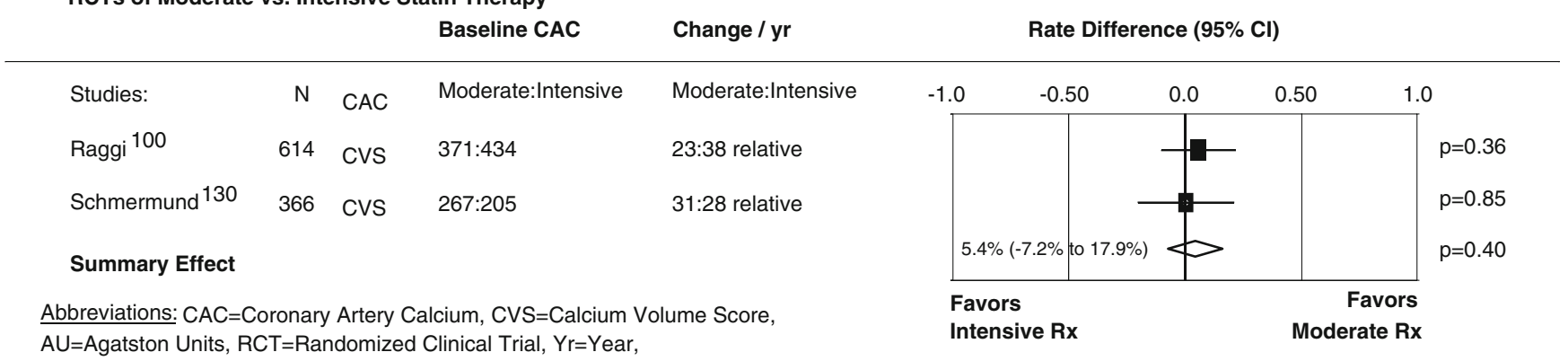

Fig. 6 Summary meta-analysis of randomized control trials (RCT) on the effect of statin therapy (Rx) on CAC progression 
$>15 \% /$ year change in CAC score had 66 and $97 \%$ MI-free survival, respectively, at 6 years $(P<0.0001)$. Patients who exhibited significant progression from their index CT ( $\geq 15 \%$ /year) and those with baseline CAC score $\geq 400$ had a more rapid presentation to acute MI occurring at 2-4 years post-testing as compared to those with CAC scores $\leq 100$ with incident MIs at over 5 years from baseline testing $(P<$ 0.0001). Thus, the baseline CAC score provides an insight into not only the expected rate of progression but also the timeline of conversion to symptomatic CAD.

\section{Summary}

The evidence is inconclusive as to what is the most accurate method to define CAC progression (percent vs. absolute vs. square root change). Further research is indicated as to documenting meaningful changes in the various scores. For the patient with an average Framingham risk score, the yearly increase in CAC score is approximately $15-20 \%$. Absolute changes are greater in patients whose baseline score exceeds 100. To date, published randomized trials have failed to demonstrate a benefit of statin therapy to attenuate CAC progression. Despite this, rapidly increasing CAC scores may be used to define higher risk patients. Further insight into the prognostic implications of serial CT examinations is warranted to further guide optimal patient management. This writing committee does not recommend the systematic performance of serial CAC scoring in every patient who has undergone a baseline $\mathrm{CT}$ and is receiving treatment for factors related to atherosclerosis. An individualized approach to assess rate of progression in specific situations may be taken into consideration.

\section{Standardization of the calcium score measured using different CT systems}

The utilization of CAC scores for outcomes data, risk stratification, and particularly, the serial assessment of patients over time demands accurate measurements. Accurate measurement of MDCT-derived CAC scores requires implementation of standardized imaging and quantification methods on many different types of commercially available MDCT systems. This formidable goal can be achieved by selecting CT parameters that fulfill minimum requirements for temporal resolution, spatial resolution, and noise and by applying a physically meaningful, calibration-based calcium quantification algorithm.

A standard for CAC quantification was recently proposed by the Physics Task Group of the International Consortium on Standardization in Cardiac CT and is reviewed here [6]. Standardized CT protocols were developed for six CT models from five manufacturers (Aquilion, Toshiba Medical Systems, Nasu, Japan; Imatron, Imatron San Francisco, CA; LightSpeed Plus, General Electric Healthcare, Milwaukee,
WI; MX8000, Philips Medical Systems, Best, The Netherlands; Volume Zoom, Siemens Medical Solutions, Erlangen, Germany; Sensation 64, Siemens) using an anthropomorphic cardiac phantom containing water and calcium inserts and capable of simulating three patient sizes. Manufacturerrecommended protocols met the minimum requirements for imaging coronary calcium with MDCT: (1) acquisition of at least four slices per rotation, (2) rotation time less than or equal to $0.5 \mathrm{~s}$, and (3) ability to reference data acquisition or reconstruction to the ECG signal. Most protocols were, however, modified to achieve a target noise level (20$23 \mathrm{HU}$ ) in the water insert for each phantom size. This primarily required determination of $\mathrm{CT}$ model- and sizespecific values for the tube current $(\mathrm{mA})$ or tube-current time product (mAs). Small, medium, and large anthropomorphic phantoms were then examined on a total of 10 different CT machines using these standardized CT protocols.

All image sets were scored using a single software package because, although not explicitly evaluated by the Consortium, differences among scoring packages are assumed to be nonnegligible (but low compared to other sources of error; see next section). To address this issue, software manufacturers were asked to modify existing algorithms according to recommendations of the Consortium. Software packages will then be validated as they become available (at least three manufacturers have incorporated the Consortium recommendations into their software at this writing).

To quantify $\mathrm{CAC}$, voxels containing calcium were first isolated from other tissue and image noise primarily by applying a standard 130-HU attenuation threshold to the reconstructed images. Agatston, volume, and mass scores were then calculated using standard quantification algorithms [6]. To obtain absolute values for calcium mass, a calibration measurement of a calcification with known hydroxyapatite (HA) density was carried out and a calibration factor determined. Because the CT number of all materials except water depends on the $\mathrm{x}$-ray spectrum, a specific calibration factor exists for each machine and each CT protocol. Work by the Physics Group of the Consortium also showed that patient size changes the X-ray spectrum and impacts the value of the calibration factor significantly. Therefore, a unique calibration factor was determined for each of three broad categories of patient sizes for each CT model and each CT protocol using the cardiac phantom's water and calcium inserts.

The mass score $\left(m_{i j}\right)$ was then computed as the product of the appropriate calibration factor $\left(c_{\mathrm{HA}}\right)$, the number of voxels containing calcium $\left(N_{\text {voxel }}\right)$, the volume of one voxel $\left(V_{\text {voxel }}\right)$, and the mean $\mathrm{CT}$ number for each lesion $\left(\overline{C T}_{i j}\right)$ :

$m_{i j}=c_{H A} \cdot N_{\text {voxel }} \cdot V_{\text {voxel }} \cdot \overline{C T}_{i j}$

The total mass score is the sum of the mass of all individual lesions.

Analysis of the mean and standard deviation of the calcium scores measured under ideal conditions from EBCT 
and MDCT systems demonstrated a coefficient of variation of $4.0 \%$ for Agatston scores, $7.9 \%$ for volume scores, and $4.9 \%$ for mass scores. The accuracy, or exact correspondence between measured and true values, could not be assessed for Agatston scores because this score represents only a mathematical construct and as such cannot be compared to a physical reference standard. However, calcium volume and mass scores could be compared to known values from the cardiac phantom. For the five MDCT systems, the total calcium mass score was within $\pm 5 \mathrm{mg}$ of the total known mass of calcium HA within the phantom $(168.2 \mathrm{mg})$. The accuracy of EBT measurement was considerably worse (mean mass score equaled $182.7 \mathrm{mg}$ ). Therefore, the increased precision of the mass score as compared with the volume score and the ability to compare the measured mass score with a known physical standard motivated the Consortium to endorse the mass score approach as the preferred method of quantifying CAC.

Additional data have been collected by the Physics Group towards optimization of the mass score. Specifically, the requirements for calculation of a calibration factor were examined. Variation in the measured calibration factor from three sizes of the anthropomorphic cardiac phantom was assessed across CT machines, time, and patient sizes. Assessment across CT machines revealed the coefficient of variation in the calibration factor was small for a specific CT manufacturer and CT model (0.13-1.6\%). Subsequent data analysis from the same CT systems over time has shown slightly higher variation for measurements made quarterly over a 4-year period from a single 16-slice CT machine (2.8-3.2\%) and over a 2-year period from a single 64-slice CT machine (2.5-3.1). The change in phantom (i.e., patient size), however, caused a much larger change in calibration factor both across CT systems (3.8-5.1\%) and over time (3.4-5.0\%). Therefore, determination of a calibration factor for a given CT machine and patient size from quarterly $\mathrm{CT}$ of an anthropomorphic phantom should be sufficiently stable over time to permit 3\% or less variation in the measurement. It has been suggested that inclusion of a calibration insert with each patient is necessary for precise measurement of a calibration factor. However, this seems unnecessary based on the low variability in calibration estimation with quarterly anthropomorphic CT.

Because of the variation in calibration measurements, particularly across patient sizes, the Consortium recommended identifying voxels containing calcium by applying a threshold based on a fixed density or concentration of calcium HA (100 mg/cc of calcium HA) rather than the traditional fixed attenuation $(130 \mathrm{HU})$ that may not provide a consistent cutoff value for calcium across examinations.

In summary, the Physics Group demonstrated that standardized protocols and algorithms can provide accurate and precise calcium mass scores in phantoms independent of MDCT model and phantom (patient) size through the use of appropriate calibration factors. Implementation of these protocols should move the field of CAC scoring closer to the realization of meaningful quantitative comparisons of
CAC scores measured over time within a patient and across patients even when imaging is performed using different MDCT models. An obvious output of the implementation of such standards should be reduced variability in CAC measurements although this remains a point of investigation.

The recommendations of the Consortium have largely been implemented by the $\mathrm{CT}$ manufacturers making adherence to these standardization procedures in clinical CT straightforward. Additional tasks beyond current practice will, however, be required including measurement of lateral skin-to-skin width at mid-liver from an anteroposterior CT radiograph ("scout" image) to assess patient size, selection of appropriate patient-size-specific $\mathrm{mA} / \mathrm{mAs}$ to achieve the noise target, and selection of appropriate patient-size-specific calibration factor to determine a density-based attenuation threshold and calculate absolute calcium mass.

The biggest obstacle to widespread use of the mass score is the paucity of data available for clinical decision-making. The CAC score is most clinically meaningful in the context of risk stratification, which requires referencing a patient's total CAC score to age- and sex-matched data. A patient is assigned to a percentile range of risk on the basis of his or her total CAC score; the percentile range is defined by flexible thresholds that take into account the independent effects of age and sex on the amount of total CAC. Most currently available databases, particularly those with a significant number of patients, contain only Agatston scores. An MDCT database founded upon standard protocols using the mass score is therefore necessary.

Implementation of a standardization procedure for the acquisition and analysis of CAC images permits the accumulation of scores from various MDCT systems in a single database. A web-based database has been developed through the efforts of the Consortium to allow collection of standardized MDCT patient risk factor and CAC data (https://clinapps.bio.ri.ccf.org/cascore/). A sufficient number of patients must be entered before assignment of a precise percentile ranking can be provided to an individual patient. Based on early data, it was determined that a total registry size of 4,000 would be sufficient to estimate the percentile ranking of future patients in the age range of $45-70$ years. To date, data from over 1,000 patients have been collected. The Writing Group supports this standardization procedure and recommends that this registry be supported.

\section{Influence of scoring parameter settings of underlying software algorithms on calcium scoring}

All scoring methods used for the determination of CAC have a common denominator. This is the algorithm used to determine which areas above the threshold HU value are calcified lesions and which can be discarded as noise. To determine this very important distinction, common algorithms are used that are influenced by a number of different parameter settings which, as shown by van Ooijen et al. in 50 patients imaged with EBT, influence the resulting CAC score 
[134]. The most common parameters are the HU threshold value, the connectivity, the lesion size threshold, and the use of interpolation. Some commercially available software packages provide the user with the parameter settings and even allow changing these parameters. Others hide the default settings, and determining the settings used can be very difficult. Mean variability can be up to $15-16$ points for the Agatston score with the largest influence coming from changing the lesion size threshold between 2 and 4 pixels. For the CVS, mean variability can be up to 20-30 points largely due to the effect of changing the lesion size threshold between 2 and 4 pixels and from turning interpolation on and off. It could well be that the effect of interpolation will be less prominent when using MDCT instead of EBT because of the use of slice overlap. There are no published data for the mass scoring method, but since this method also relies on algorithms to determine what regions are lesions and what regions are not, it is likely that similar results will be found.

In conclusion, when performing $\mathrm{CAC}$ scoring based on the volume or Agatston score, software parameter settings affect the outcome. Furthermore, the use of new software versions or other software packages and the use of data acquired in other institutes in the follow-up of patients could also affect the measured progression or regression of CAC because of different parameter settings. These data show, therefore, that not only standardization of CT protocols is obligatory, but CAC scoring parameters also need to be standardized. Further research is required to determine whether using phantom data or test patient datasets can help standardize settings across software and help select the appropriate settings of a certain software package when they are unclear.

\section{Radiation exposure}

A broad implementation of CAC screening may be limited by factors such as cost, patient access, and demonstration of altered medical outcomes. In addition, risks associated with the use of ionizing radiation must be taken into account, especially for younger or female patients or when considering additional radiological tests such as $\mathrm{CT}$ and MPI. CAC screening delivers a relatively low radiation dose (effective dose of $0.7 \mathrm{mSv}$ with EBT and 1.0-4.1 mSv with MDCT) [135], while coronary CT angiography (outside of the scope of this writing) delivers somewhat increased levels of radiation dose (effective dose of 9.4-14.8 mSv) [136]. The dose to any one individual depends both on the imaging protocol used and the patient's body habitus. The radiation exposure provided by CAC screening is substantially lower than that of MPI studies (effective dose range of 13-16 mSv), especially those conducted using thallium-201 or dual isotope techniques (effective dose of $27.3 \mathrm{mSv}$ ) [137] or invasive diagnostic coronary catheterization (effective dose of 3-10 mSv) [138].

Much of our knowledge on the carcinogenic effects of low doses of radiation (whole-body exposures of 5-150 mSv) derives from follow-up data on the survivors of the atomic bombings in Japan. Although quite small, there appears to be an increase in incidence of cancer in subjects exposed to low doses of radiation, especially in children because of the higher radiation sensitivity and the longer available time for cancer development after exposure. Using the linear nonthreshold model of radiation-induced risk and the organ-specific risk from Biological Effects of Ionizing Radiation (BEIR VII) [139], the data presented by Einstein et al. [140] can be linearly scaled to predict the lifetime risk of cancer.

Assuming a factor of 10 reduction of dose from a coronary CTA exam, the lifetime risk of cancer for a CAC CT in a 50year-old individual is $0.04 \%$ for a man and $0.12 \%$ for a woman. To properly interpret these data, the individual's complete risk profile must be considered, including the background risk of cancer incidence in the general population and any individualspecific risks such as diabetes, high blood pressure, or a family history of cancer or heart disease. According to statistics from the American Cancer Society, the lifetime risk of cancer at any site is $45 \%$ for men and $38 \%$ for women; the respective death rates are 23 and $20 \%$ [141]. Thus, taking into account the patient's specific medical risks, particularly of CAD, and the background population risks, the additive cancer risk from a CAC exam is negligible, provided that some benefit may be gained from the examination. Thus, this committee of experts does not support the application of CAC screening to individuals at low risk of $\mathrm{CAD}$, where medical benefit is not expected. For individuals at intermediate risk of CAD, the small statistical risk of cancer induction and death is very low relative to the patient's complete risk profile. In these patients, the potential benefit to the patient from knowledge obtained in the CAC exam greatly exceeds the small potential risk of cancer and the use of CAC screening is recommended in several clinical scenarios.

Further, in contrast to alarming media reports regarding the risks associated with ionizing radiation, the radiation biology and epidemiology community is divided as to the actual degree of risk at the low doses associated with medical imaging examinations. Considering the error bars associated with the data from the Japanese bomb survivors, the difficulty in transferring risk estimates between population cohorts and irradiation dose rates and types (high vs. low dose rates, whole body vs. partial body exposures, etc.), and the conflicting reports from medically exposed populations that show no increase in risk at medical-imaging dose levels, it is the official position of the Health Physics Society that meaningful risk estimates are not possible below effective doses of $100 \mathrm{mSv}$ [142]. Thus, CAC exams, with effective doses of $1-4 \mathrm{mSv}$, may in fact be associated with no additional risk and hence should not be avoided when information important to the patient's medical management may be obtained.

\section{Conclusions}

The writing committee would like to summarize in a series of conceptual points the evidence discussed herein as follows. 
- We know and support the conclusion that:

- CAC is a good predictor of events in Caucasians and adds incremental prognostic value to risk factors in intermediate risk populations.

- There is significant variability among ethnicities in the prevalence and extent of coronary calcium.

- Absence of CAC is associated with very low event rates in most risk categories.

- Rapid CAC progression is associated with higher risk of events.

- CAC is a strong predictor of events in end-stage renal disease.

- A zero calcium score is associated with a very low prevalence of ischemia on functional stress testing and obstructive disease on angiography.

- We are beginning to understand that:

- CAC may have good predictive value in the elderly, diabetic patients, and patients of different ethnic background.

- CAC scores can be used to predict presence of obstructive CAD, but despite a high sensitivity, this tool demonstrates a low specificity; hence the main utilization of CAC should be assessment of risk of cardiovascular events rather than the detection of severe CAD.

- We still need to prove that:

- We can alter CAC progression with medical interventions.

- Altering CAC progression with medical interventions impacts patients' outcome.

- We may not need to treat patients with risk factors in the absence of CAC.
Finally, standardized procedures for both image acquisition and CAC scoring should be followed so that we might best advance our knowledge using MDCT.

\section{Disclosure of support}

\begin{tabular}{ll}
\hline Author & Disclosure of Support \\
\hline SSH & None \\
WAK & Siemens Medical Solutions \\
CHM & Bayer Healthcare, Siemens Medical Solutions, \\
& RTI Electronics, Inc \\
SM & None \\
MO & None \\
PMAO & None \\
PR & None \\
AES & Astellas, Siemens Medical Solutions \\
LJS & None \\
WS & None \\
AJT & None \\
RV & None \\
LW & None \\
\hline
\end{tabular}

Open Access This article is distributed under the terms of the Creative Commons Attribution Noncommercial License which permits any noncommercial use, distribution, and reproduction in any medium, provided the original author(s) and source are credited.

\section{References}

1. O'Rourke RA, Brundage BH, Froelicher VF et al (2000) American College of Cardiology/American Heart Association Expert Consensus document on electronbeam computed tomography for the diagnosis and prognosis of coronary artery disease. Circulation 102:126-140
2. Wexler L, Brundage B, Crouse J et al (1996) Coronary artery calcification: pathophysiology, epidemiology, imaging methods, and clinical implications. A statement for health professionals from the American Heart Association. Writing Group. Circulation 94:1175-1192

3. Budoff MJ, Achenbach S, Blumenthal RS et al (2006) Assessment of coronary artery disease by cardiac computed tomography: a scientific statement from the American Heart Association Committee on Cardiovascular Imaging and Intervention, Council on Cardiovascular Radiology and Intervention, and Committee on Cardiac Imaging, Council on Clinical Cardiology. Circulation 114:1761-1791
4. Greenland $\mathrm{P}$, Bonow RO, Brundage $\mathrm{BH}$ et al (2007) ACCF/AHA 2007 clinical expert consensus document on coronary artery calcium scoring by computed tomography in global cardiovascular risk assessment and in evaluation of patients with chest pain: a report of the American College of Cardiology Foundation Clinical Expert Consensus Task Force (ACCF/AHA Writing Committee to Update the 2000 Expert Consensus Document on Electron Beam Computed Tomography). Circulation 115:402-426 
5. Hendel RC, Patel MR, Kramer CM et al (2006) ACCF/ACR/SCCT/SCMR/ ASNC/NASCI/SCAI/SIR 2006 appropriateness criteria for cardiac computed tomography and cardiac magnetic resonance imaging: a report of the American College of Cardiology Foundation Quality Strategic Directions Committee Appropriateness Criteria Working Group, American College of Radiology, Society of Cardiovascular Computed Tomography, Society for Cardiovascular Magnetic Resonance, American Society of Nuclear Cardiology, North American Society for Cardiac Imaging, Society for Cardiovascular Angiography and Interventions, and Society of Interventional Radiology. J Am Coll Cardiol 48:1475-1497

6. McCollough $\mathrm{CH}$, Ulzheimer S, Halliburton SS et al (2007) Coronary artery calcium: a multi-institutional, multimanufacturer international standard for quantification at cardiac CT. Radiology 243:527-538

7. Becker CR, Kleffel T, Crispin A et al (2001) Coronary artery calcium measurement: agreement of multirow detector and electron beam CT. AJR Am J Roentgenol 176:1295-1298

8. Carr JJ, Crouse JR 3rd, Goff DC Jr (2000) Evaluation of subsecond gated helical CT for quantification of coronary artery calcium and comparison with electron beam CT. AJR Am J Roentgenol 174:915-921

9. Daniell AL, Wong ND, Friedman JD et al (2005) Concordance of coronary artery calcium estimates between MDCT and electron beam tomography. AJR Am J Roentgenol 185:1542-1545

10. Detrano RC, Anderson M, Nelson J et al (2005) Coronary calcium measurements: effect of CT scanner type and calcium measure on rescan reproducibility-MESA study. Radiology 236:477-484

11. Horiguchi J, Yamamoto H, Akiyama Y et al (2004) Coronary artery calcium scoring using 16-MDCT and a retrospective ECG-gating reconstruction algorithm. AJR Am J Roentgenol 183:103-108
12. Knez A, Becker C, Becker A et al (2002) Determination of coronary calcium with multi-slice spiral computed tomography: a comparative study with electron-beam CT. Int J Cardiovasc Imaging 18:295-303

13. Greuter MJ, Dijkstra H, Groen JM et al (2007) 64 slice MDCT generally underestimates coronary calcium scores as compared to EBT: a phantom study. Med Phys 34:3510-3519

14. Ulzheimer S, Kalender WA (2003) Assessment of calcium scoring performance in cardiac computed tomography. Eur Radiol 13:484-497

15. Jain T, Peshock R, McGuire DK et al (2004) African Americans and Caucasians have a similar prevalence of coronary calcium in the Dallas Heart Study. J Am Coll Cardiol 44:10111017

16. Bild DE, Detrano R, Peterson D et al (2005) Ethnic differences in coronary calcification: the Multi-Ethnic Study of Atherosclerosis (MESA). Circulation 111:1313-1320

17. Oei HH, Vliegenthart R, Hofman A et al (2004) Risk factors for coronary calcification in older subjects. The Rotterdam Coronary Calcification Study. Eur Heart J 25:48-55

18. Vliegenthart R, Oudkerk M, Hofman A et al (2005) Coronary calcification improves cardiovascular risk prediction in the elderly. Circulation 112:572-577

19. Arad Y, Spadaro LA, Goodman K et al (2000) Prediction of coronary events with electron beam computed tomography. J Am Coll Cardiol 36:12531260

20. Greenland P, LaBree L, Azen SP et al (2004) Coronary artery calcium score combined with Framingham score for risk prediction in asymptomatic individuals. JAMA 291:210-215

21. Kondos GT, Hoff JA, Sevrukov A et al (2003) Electron-beam tomography coronary artery calcium and cardiac events: a 37-month follow-up of 5635 initially asymptomatic low- to intermediate-risk adults. Circulation 107:2571-2576

22. LaMonte MJ, FitzGerald SJ, Church TS et al (2005) Coronary artery calcium score and coronary heart disease events in a large cohort of asymptomatic men and women. Am J Epidemiol 162:421429

23. Taylor AJ, Bindeman J, Feuerstein I et al (2005) Coronary calcium independently predicts incident premature coronary heart disease over measured cardiovascular risk factors: mean threeyear outcomes in the Prospective Army Coronary Calcium (PACC) project. J Am Coll Cardiol 46:807-814
24. Raggi P, Callister TQ, Cooil B et al (2000) Identification of patients at increased risk of first unheralded acute myocardial infarction by electron-beam computed tomography. Circulation 101:850-855

25. Pletcher MJ, Tice JA, Pignone $M$ et al (2004) Using the coronary artery calcium score to predict coronary heart disease events: a systematic review and meta-analysis. Arch Intern Med 164:1285-1292

26. Haberl R, Becker A, Leber A et al (2001) Correlation of coronary calcification and angiographically documented stenoses in patients with suspected coronary artery disease: results of 1,764 patients. J Am Coll Cardiol 37:451457

27. Knez A, Becker A, Leber A et al (2004) Relation of coronary calcium scores by electron beam tomography to obstructive disease in 2,115 symptomatic patients. Am J Cardiol 93:1150-1152

28. Budoff MJ, Diamond GA, Raggi P et al (2002) Continuous probabilistic prediction of angiographically significant coronary artery disease using electron beam tomography. Circulation 105:1791-1796

29. Wilson PW, D'Agostino RB, Levy D et al (1998) Prediction of coronary heart disease using risk factor categories. Circulation 97:1837-1847

30. Assmann G, Cullen P, Schulte H (2002) Simple scoring scheme for calculating the risk of acute coronary events based on the 10-year follow-up of the prospective cardiovascular Munster (PROCAM) study. Circulation 105:310-315

31. Conroy RM, Pyorala K, Fitzgerald AP et al (2003) Estimation of ten-year risk of fatal cardiovascular disease in Europe: the SCORE project. Eur Heart J 24:987-1003

32. Graham I, Atar D, Borch-Johnsen K et al (2007) European guidelines on cardiovascular disease prevention in clinical practice: executive summary. Fourth Joint Task Force of the European Society of Cardiology and other societies on cardiovascular disease prevention in clinical practice (constituted by representatives of nine societies and by invited experts). Eur J Cardiovasc Prev Rehabil 14 Suppl 2: E1-E40 
33. Smith SC Jr, Amsterdam E, Balady GJ et al (2000) Prevention Conference V: beyond secondary prevention: identifying the high-risk patient for primary prevention: tests for silent and inducible ischemia: Writing Group II. Circulation 101:E12-E16

34. Grundy SM, Cleeman JI, Merz CN et al (2004) Implications of recent clinical trials for the National Cholesterol Education Program Adult Treatment Panel III Guidelines. J Am Coll Cardiol 44:720-732

35. Patel MR, Spertus JA, Brindis RG et al (2005) ACCF proposed method for evaluating the appropriateness of cardiovascular imaging. J Am Coll Cardiol 46:1606-1613

36. Arad Y, Goodman KJ, Roth $\mathrm{M}$ et al (2005) Coronary calcification, coronary disease risk factors, C-reactive protein, and atherosclerotic cardiovascular disease events: the St. Francis Heart Study. J Am Coll Cardiol 46:158-165

37. Erbel R, Mohlenkamp S, Lehmann N et al (2008) Sex related cardiovascular risk stratification based on quantification of atherosclerosis and inflammation. Atherosclerosis 197:662-672

38. Gibbons RJ, Balady GJ, Bricker JT et al (2002) ACC/AHA 2002 guideline update for exercise testing: summary article: a report of the American College of Cardiology/American Heart Association Task Force on Practice Guidelines (Committee to Update the 1997 Exercise Testing Guidelines). Circulation 106:1883-1892

39. Fleischmann KE, Hunink MG, Kuntz $\mathrm{KM}$ et al (2002) Exercise echocardiography or exercise SPECT imaging? A meta-analysis of diagnostic test performance. J Nucl Cardiol 9:133-134

40. Berman DS, Wong ND, Gransar H et al (2004) Relationship between stressinduced myocardial ischemia and atherosclerosis measured by coronary calcium tomography. J Am Coll Cardiol 44:923-930

41. Georgiou D, Budoff MJ, Kaufer E et al (2001) Screening patients with chest pain in the emergency department using electron beam tomography: a follow-up study. J Am Coll Cardiol 38:105-110

42. Shaw LJ, Raggi P, Callister TQ et al (2006) Prognostic value of coronary artery calcium screening in asymptomatic smokers and non-smokers. Eur Heart J 27:968-975
43. Geluk CA, Dikkers R, Perik PJ et al (2008) Measurement of coronary calcium scores by electron beam computed tomography or exercise testing as initial diagnostic tool in low-risk patients with suspected coronary artery disease. Eur Radiol 18:244-252

44. Raggi $\mathrm{P}$, Callister TQ, Cooil B et al (2000) Evaluation of chest pain in patients with low to intermediate pretest probability of coronary artery disease by electron beam computed tomography. Am J Cardiol 85:283-288

45. Kalia NK, Miller LG, Nasir K et al (2006) Visualizing coronary calcium is associated with improvements in adherence to statin therapy. Atherosclerosis 185:394-399

46. Doherty TM, Tang W, Detrano RC (1999) Racial differences in the significance of coronary calcium in asymptomatic black and white subjects with coronary risk factors. J Am Coll Cardiol 34:787-794

47. Santos RD, Nasir K, Rumberger JA et al (2006) Difference in atherosclerosis burden in different nations and continents assessed by coronary artery calcium. Atherosclerosis 187:378-384

48. Detrano R, Carr JC, Wong ND et al (2007) Coronary calcium predicts nearterm coronary heart disease events in major American ethnic groups: the Multi-Ethnic Study of Atherosclerosis. J Am Coll Cardiol 811-813

49. Nasir K, Shaw LJ, Liu ST et al (2007) Ethnic differences in the prognostic value of coronary artery calcification for all-cause mortality. J Am Coll Cardiol 50:953-960

50. Sirineni GK, Raggi P, Shaw LJ et al (2008) Calculation of coronary age using calcium scores in multiple ethnicities. Int $\mathrm{J}$ Cardiovasc Imaging 24:107-111

51. Anderson KM, Castelli WP, Levy D (1987) Cholesterol and mortality. 30 years of follow-up from the Framingham study. JAMA 257:2176-2180

52. Glynn RJ, Field TS, Rosner B et al (1995) Evidence for a positive linear relation between blood pressure and mortality in elderly people. Lancet 345:825-829

53. Kannel WB, D'Agostino RB, Silbershatz H (1997) Blood pressure and cardiovascular morbidity and mortality rates in the elderly. Am Heart J 134:758-763

54. Thompson GR, Partridge J (2004) Coronary calcification score: the coronary-risk impact factor. Lancet 363:557-559

55. Abbott RD, Ueshima H, Masaki KH et al (2007) Coronary artery calcification and total mortality in elderly men. J Am Geriatr Soc 55:1948-1954
56. Newman AB, Naydeck BL, Ives DG et al (2008) Coronary artery calcium, carotid artery wall thickness, and cardiovascular disease outcomes in adults 70 to 99 years old. Am J Cardiol 101:186-192

57. Budoff MJ, Shaw LJ, Liu ST et al (2007) Long-term prognosis associated with coronary calcification: observations from a registry of 25,253 patients. J Am Coll Cardiol 49: $1860-1870$

58. Wong ND, Sciammarella MG, Polk D et al (2003) The metabolic syndrome, diabetes, and subclinical atherosclerosis assessed by coronary calcium. J Am Coll Cardiol 41:1547-1553

59. Schurgin S, Rich S, Mazzone T (2001) Increased prevalence of significant coronary artery calcification in patients with diabetes. Diabetes Care 24:335338

60. Khaleeli E, Peters SR, Bobrowsky K et al (2001) Diabetes and the associated incidence of subclinical atherosclerosis and coronary artery disease: Implications for management. Am Heart J 141:637-644

61. Mielke CH, Shields JP, Broemeling LD (2001) Coronary artery calcium, coronary artery disease, and diabetes. Diabetes Res Clin Pract 53:55-61

62. Hoff JA, Quinn L, Sevrukov A et al (2003) The prevalence of coronary artery calcium among diabetic individuals without known coronary artery disease. J Am Coll Cardiol 41:1008-1012

63. Olson JC, Edmundowicz D, Becker DJ et al (2000) Coronary calcium in adults with type 1 diabetes: a stronger correlate of clinical coronary artery disease in men than in women. Diabetes 49:1571-1578

64. Wong ND, Rozanski A, Gransar $\mathrm{H}$ et al (2005) Metabolic syndrome and diabetes are associated with an increased likelihood of inducible myocardial ischemia among patients with subclinical atherosclerosis. Diabetes Care 28:1445-1450

65. Anand DV, Lim E, Hopkins D et al (2006) Risk stratification in uncomplicated type 2 diabetes: prospective evaluation of the combined use of coronary artery calcium imaging and selective myocardial perfusion scintigraphy. Eur Heart J 27:713-721

66. Qu W, Le TT, Azen SP et al (2003) Value of coronary artery calcium scanning by computed tomography for predicting coronary heart disease in diabetic subjects. Diabetes Care 26:905-910 
67. Raggi P, Shaw LJ, Berman DS et al (2004) Prognostic value of coronary artery calcium screening in subjects with and without diabetes. J Am Coll Cardiol 43:1663-1669

68. Russo D, Palmiero G, De Blasio AP et al (2004) Coronary artery calcification in patients with CRF not undergoing dialysis. Am J Kidney Dis 44:10241030

69. Bursztyn M, Motro M, Grossman E et al (2003) Accelerated coronary artery calcification in mildly reduced renal function of high-risk hypertensives: a 3-year prospective observation. J Hypertens 21:1953-1959

70. Sigrist M, Bungay P, Taal MW et al (2006) Vascular calcification and cardiovascular function in chronic kidney disease. Nephrol Dial Transplant 21:707-714

71. Block GA, Raggi P, Bellasi A et al (2007) Mortality effect of coronary calcification and phosphate binder choice in incident hemodialysis patients. Kidney Int 71:438-441

72. Raggi P, Boulay A, Chasan-Taber S et al (2002) Cardiac calcification in adult hemodialysis patients. A link between end-stage renal disease and cardiovascular disease? J Am Coll Cardiol 39:695-701

73. Braun J, Oldendorf M, Moshage W et al (1996) Electron beam computed tomography in the evaluation of cardiac calcification in chronic dialysis patients. Am J Kidney Dis 27:394-401

74. Goodman WG, Goldin J, Kuizon BD et al (2000) Coronary-artery calcification in young adults with end-stage renal disease who are undergoing dialysis. $\mathrm{N}$ Engl J Med 342:1478-1483

75. Oh J, Wunsch R, Turzer M et al (2002) Advanced coronary and carotid arteriopathy in young adults with childhoodonset chronic renal failure. Circulation 106:100-105

76. Wang AY, Wang M, Woo J et al (2003) Cardiac valve calcification as an important predictor for all-cause mortality and cardiovascular mortality in longterm peritoneal dialysis patients: a prospective study. J Am Soc Nephrol 14:159-168

77. Chertow GM, Raggi P, Chasan-Taber S et al (2004) Determinants of progressive vascular calcification in haemodialysis patients. Nephrol Dial Transplant 19:1489-1496
78. Chertow GM, Burke SK, Raggi P (2002) Sevelamer attenuates the progression of coronary and aortic calcification in hemodialysis patients. Kidney Int 62:245-252

79. Guerin AP, London GM, Marchais SJ et al (2000) Arterial stiffening and vascular calcifications in end-stage renal disease. Nephrol Dial Transplant 15:1014-1021

80. Block GA, Spiegel DM, Ehrlich J et al (2005) Effects of Sevelamer and calcium on coronary artery calcification in patients new to hemodialysis. Kidney Int 68:1815-1824

81. Matsuoka M, Iseki $\mathrm{K}$, Tamashiro $\mathrm{M}$ et al (2004) Impact of high coronary artery calcification score (CACS) on survival in patients on chronic hemodialysis. Clin Exp Nephrol 8:54-58

82. Shaw LJ, Raggi P, Schisterman E et al (2003) Prognostic value of cardiac risk factors and coronary artery calcium screening for all-cause mortality. Radiology 228:826-833

83. Church TS, Levine BD, McGuire DK et al (2007) Coronary artery calcium score, risk factors, and incident coronary heart disease events. Atherosclerosis 190:224-231

84. Raggi P, Shaw LJ, Berman DS et al (2004) Gender-based differences in the prognostic value of coronary calcification. J Womens Health (Larchmt) $13: 273-283$

85. Bellasi A, Lacey C, Taylor AJ et al (2007) Comparison of prognostic usefulness of coronary artery calcium in men versus women (results from a meta- and pooled analysis estimating all-cause mortality and coronary heart disease death or myocardial infarction). Am J Cardiol 100:409-414

86. Becker A, Leber A, White CW et al (2007) Multislice computed tomography for determination of coronary artery disease in a symptomatic patient population. Int J Cardiovasc Imaging 23:361-367

87. Cheng VY, Lepor NE, Madyoon $\mathrm{H}$ et al (2007) Presence and severity of noncalcified coronary plaque on 64-slice computed tomographic coronary angiography in patients with zero and low coronary artery calcium. Am J Cardiol 99:1183-1186

88. Leschka S, Scheffel H, Desbiolles L et al (2007) Combining dual-source computed tomography coronary angiography and calcium scoring: added value for the assessment of coronary artery disease. Heart Nov 21 [Epub ahead of print]
89. Rubinshtein R, Gaspar T, Halon DA et al (2007) Prevalence and extent of obstructive coronary artery disease in patients with zero or low calcium score undergoing 64-slice cardiac multidetector computed tomography for evaluation of a chest pain syndrome. Am J Cardiol 99:472-475

90. Lamont DH, Budoff MJ, Shavelle DM et al (2002) Coronary calcium scanning adds incremental value to patients with positive stress tests. Am Heart J 143:861-867

91. Stillman AE, Oudkerk M, Ackerman M et al (2007) Use of multidetector computed tomography for the assessment of acute chest pain: a consensus statement of the North American Society of Cardiac Imaging and the European Society of Cardiac Radiology. Eur Radiol 17:2196-2207

92. Stillman AE, Oudkerk M, Ackerman M et al (2007) Use of multidetector computed tomography for the assessment of acute chest pain: a consensus statement of the North American Society of Cardiac Imaging and the European Society of Cardiac Radiology. Int J Cardiovasc Imaging 23:415-427

93. Laudon DA, Vukov LF, Breen JF et al (1999) Use of electron-beam computed tomography in the evaluation of chest pain patients in the emergency department. Ann Emerg Med 33:15-21

94. McLaughlin VV, Balogh T, Rich S (1999) Utility of electron beam computed tomography to stratify patients presenting to the emergency room with chest pain. Am J Cardiol 84:327-328

95. Andrews TC (2000) Electron-beam computed tomography in the evaluation of patients with chest pain. Am J Cardiol 85:386-387

96. Villines TC, Taylor AJ (2005) Noninvasive atherosclerosis imaging: use to assess response to novel or combination lipid therapies. Curr Drug Targets Cardiovasc Haematol Disord 5:557564

97. Raggi P, Callister TQ, Shaw LJ (2004) Progression of coronary artery calcium and risk of first myocardial infarction in patients receiving cholesterol-lowering therapy. Arterioscler Thromb Vasc Biol 24:1272-1277

98. Raggi P, Cooil B, Ratti C et al (2005) Progression of coronary artery calcium and occurrence of myocardial infarction in patients with and without diabetes mellitus. Hypertension 46:238-243 
99. Raggi P, Cooil B, Shaw LJ et al (2003) Progression of coronary calcium on serial electron beam tomographic scanning is greater in patients with future myocardial infarction. Am J Cardiol 92:827-829

100. Raggi P, Davidson M, Callister TQ et al (2005) Aggressive versus moderate lipid-lowering therapy in hypercholesterolemic postmenopausal women: Beyond Endorsed Lipid Lowering with EBT Scanning (BELLES). Circulation 112:563-571

101. Raggi P, Taylor A, Fayad Z et al (2005) Atherosclerotic plaque imaging: contemporary role in preventive cardiology. Arch Intern Med $165 \cdot 2345-2353$

102. Taylor A, Shaw LJ, Fayad Z et al (2005) Tracking atherosclerosis regression: a clinical tool in preventive cardiology. Atherosclerosis 180:1-10

103. Therasse P, Arbuck SG, Eisenhauer EA et al (2000) New guidelines to evaluate the response to treatment in solid tumors. European Organization for Research and Treatment of Cancer, National Cancer Institute of the United States, National Cancer Institute of Canada. J Natl Cancer Inst 92:205216

104. Budoff MJ, Raggi P (2001) Coronary artery disease progression assessed by electron-beam computed tomography. Am J Cardiol 88:46E-50E

105. Lu B, Zhuang N, Mao SS et al (2002) EKG-triggered CT data acquisition to reduce variability in coronary arterial calcium score. Radiology 224:838844

106. Mao S, Bakhsheshi H, Lu B et al (2001) Effect of electrocardiogram triggering on reproducibility of coronary artery calcium scoring. Radiology 220:707-711

107. Hoffmann U, Siebert U, Bull-Stewart A et al (2006) Evidence for lower variability of coronary artery calcium mineral mass measurements by multidetector computed tomography in a community-based cohort-consequences for progression studies. Eur J Radiol 57:396-402

108. Hokanson JE, MacKenzie T, Kinney G et al (2004) Evaluating changes in coronary artery calcium: an analytic method that accounts for interscan variability. AJR Am J Roentgenol 182:1327-1332
109. Groen JM, Greuter MJ, Schmidt B et al (2007) The influence of heart rate, slice thickness, and calcification density on calcium scores using 64-slice multidetector computed tomography: a systematic phantom study. Invest Radiol 42:848-855

110. Horiguchi J, Matsuura N, Yamamoto $\mathrm{H}$ et al (2008) Variability of repeated coronary artery calcium measurements by $1.25-\mathrm{mm}$ - and $2.5-\mathrm{mm}$-thickness images on prospective electrocardiograph-triggered 64-slice CT. Eur Radiol 18:209-216

111. Horiguchi J, Yamamoto H, Hirai N et al (2006) Variability of repeated coronary artery calcium measurements on low-dose ECG-gated 16-MDCT. AJR Am J Roentgenol 187:W1-W6

112. Becker A, Leber A, von Ziegler F et al (2007) Comparison of progression of coronary calcium in postmenopausal women on versus not on estrogen/ progestin therapy. Am J Cardiol 99:374-378

113. Budoff MJ, Chen GP, Hunter CJ et al (2005) Effects of hormone replacement on progression of coronary calcium as measured by electron beam tomography. J Womens Health (Larchmt) 14:410-417

114. Gopal A, Nasir K, Liu ST et al (2007) Coronary calcium progression rates with a zero initial score by electron beam tomography. Int J Cardiol 117:227-231

115. Hsia J, Klouj A, Prasad A et al (2004) Progression of coronary calcification in healthy postmenopausal women. BMC Cardiovasc Disord 4:21

116. Rasouli ML, Nasir K, Blumenthal RS et al (2005) Plasma homocysteine predicts progression of atherosclerosis. Atherosclerosis 181:159-165

117. Shemesh J, Apter S, Stolero D et al (2001) Annual progression of coronary artery calcium by spiral computed tomography in hypertensive patients without myocardial ischemia but with prominent atherosclerotic risk factors, in patients with previous angina pectoris or healed acute myocardial infarction, and in patients with coronary events during follow-up. Am J Cardiol 87:1395-1397

118. Sutton-Tyrrell K, Kuller LH, Edmundowicz D et al (2001) Usefulness of electron beam tomography to detect progression of coronary and aortic calcium in middle-aged women. Am J Cardiol 87:560-564

119. Yoon HC, Emerick AM, Hill JA et al (2002) Calcium begets calcium: progression of coronary artery calcification in asymptomatic subjects. Radiology 224:236-241
120. Sevrukov AB, Bland JM, Kondos GT (2005) Serial electron beam CT measurements of coronary artery calcium: has your patient's calcium score actually changed? AJR Am J Roentgenol 185:1546-1553

121. Cassidy AE, Bielak LF, Zhou Y et al (2005) Progression of subclinical coronary atherosclerosis: does obesity make a difference? Circulation 111:1877-1882

122. Kawakubo M, LaBree L, Xiang M et al (2005) Race-ethnic differences in the extent, prevalence, and progression of coronary calcium. Ethn Dis 15:198-204

123. Kronmal RA, McClelland RL, Detrano $R$ et al (2007) Risk factors for the progression of coronary artery calcification in asymptomatic subjects: results from the Multi-Ethnic Study of Atherosclerosis (MESA). Circulation 115:2722-2730

124. Mehrotra R, Budoff M, Christenson P et al (2004) Determinants of coronary artery calcification in diabetics with and without nephropathy. Kidney Int 66:2022-2031

125. Snell-Bergeon JK, Hokanson JE, Jensen L et al (2003) Progression of coronary artery calcification in type 1 diabetes: the importance of glycemic control. Diabetes Care 26:2923-2928

126. Budoff MJ, Lane KL, Bakhsheshi $\mathrm{H}$ et al (2000) Rates of progression of coronary calcium by electron beam tomography. Am J Cardiol 86:8-11

127. Budoff MJ, Yu D, Nasir K et al (2005) Diabetes and progression of coronary calcium under the influence of statin therapy. Am Heart J 149:695-700

128. Callister TQ, Raggi P, Cooil B et al (1998) Effect of HMG-CoA reductase inhibitors on coronary artery disease as assessed by electron-beam computed tomography. N Engl J Med 339:19721978

129. Achenbach S, Ropers D, Pohle K et al (2002) Influence of lipid-lowering therapy on the progression of coronary artery calcification: a prospective evaluation. Circulation 106:1077-1082

130. Schmermund A, Achenbach S, Budde $T$ et al (2006) Effect of intensive versus standard lipid-lowering treatment with atorvastatin on the progression of calcified coronary atherosclerosis over 12 months: a multicenter, randomized, double-blind trial. Circulation 113:427437 
131. Harman SM, Brinton EA, Cedars M et al (2005) KEEPS: The Kronos Early Estrogen Prevention Study. Climacteric 8:3-12

132. Kuller LH, Kriska AM, Kinzel LS et al (2007) The clinical trial of Women On the Move through Activity and Nutrition (WOMAN) study. Contemp Clin Trials 28:370-381

133. Manson JE, Allison MA, Rossouw JE et al (2007) Estrogen therapy and coronary-artery calcification. N Engl J Med 356:2591-2602
134. van Ooijen PM, Vliegenthart R, Witteman JC et al (2005) Influence of scoring parameter settings on Agatston and volume scores for coronary calcification. Eur Radiol 15:102-110

135. Morin RL, Gerber TC, McCollough CH (2003) Radiation dose in computed tomography of the heart. Circulation 107:917-922

136. Hausleiter J, Meyer T, Hadamitzky M et al (2006) Radiation dose estimates from cardiac multislice computed tomography in daily practice: impact of different scanning protocols on effective dose estimates. Circulation 113:1305-1310

137. Thompson RC, Cullom SJ (2006) Issues regarding radiation dosage of cardiac nuclear and radiography procedures. J Nucl Cardiol 13:19-23

138. Einstein AJ, Moser KW, Thompson $\mathrm{RC}$ et al (2007) Radiation dose to patients from cardiac diagnostic imaging. Circulation 116:1290-1305
139. National Research Council (U.S.) Committee to Assess Health Risks from Exposure to Low Level of Ionizing Radiation (2006) Health risks from exposure to low levels of ionizing radiation: BEIR VII Phase 2. National Academies Press, Washington, DC

140. Einstein AJ, Henzlova MJ, Rajagopalan S (2007) Estimating risk of cancer associated with radiation exposure from 64-slice computed tomography coronary angiography. JAMA 298:317-323

141. Jemal A, Siegel R, Ward E et al (2007) Cancer statistics, 2007. CA Cancer J Clin 57:43-66

142. Health Physics Society (2004) Radiation risk in perspective. Available at: http://hps.org/documents/risk_ps0101.pdf. Accessed March 18, 2008 